\title{
Analysis of Water Waves Passing Over a Submerged Rectangular Dike
}

\author{
I-Chi Chan ${ }^{1}$; Liang-Hsiung Huang ${ }^{2}$; and Ping-Cheng Hsieh ${ }^{3}$
}

\begin{abstract}
The analytic solutions of inviscid and viscous water waves passing over a submerged rectangular dike are investigated. Owing to the fact that the orthogonality of eigenfunctions is invalid for viscous wave problem, two newly developed orthogonal inner products are applied to reduce the mathematical difficulty of viscous wave problem. Both inviscid and viscous water wave solutions are obtained under the assumption of linear water wave without separation. It shows that two solutions have no significant kinematic difference but the viscous contribution of dynamic effect is not negligible. Beside giving a better theoretical approach, which reduces the error of the conventional minimal squares method, the result of the present analytical solution can be used to quantitatively evaluate the correctness of experiments and also provides helpful information such as near wall boundary layer thickness and oscillating free surface for computational use.
\end{abstract}

DOI: $10.1061 /(A S C E) 0733-9399(2003) 129: 6(613)$

CE Database subject headings: Water waves; Dikes; Viscous flow; Submerging.

\section{Introduction}

The problems of propagation of water waves over a submerged obstacle have been studied theoretically by many investigators within the framework of linearized potential theory. Since the submerged structures are usually used to reduce the transmission wave, these former studies are mainly concerned with determining the reflection and transmission properties for a given incident wave. Examples of the well-known investigations are Newman (1965), Miles (1967), and Mei and Black (1969).

The above researches have good performances under the assumption that the fluid is ideal. However, the nature of the flow around the submerged obstacle is expected to have a viscous effect. According to the constitutive law of Newtonian fluid, the viscous stress is caused since the velocity gradient occurs by the sudden change of the vertical cross section of water due to the submerged obstacle. Therefore, dynamic effects can no longer be predicted accurately by potential theory. It would be interesting to investigate the wave characteristics over the submerged obstacle by using the viscous flow approach, but this kind of analytical solution, to the authors' knowledge, is seldom found due to mathematical difficulties. The orthogonal solutions of potential flow no longer existed because of complex wave numbers caused by viscous effect. Lo and Lee (2000) adopt the minimal squares method

\footnotetext{
${ }^{1}$ Dept. of Civil Engineering, National Taiwan Univ., Taipei, Taiwan, ROC.

${ }^{2}$ Dept. of Civil Engineering, National Taiwan Univ., Taipei, Taiwan, ROC.

${ }^{3}$ Dept. of Soil and Water Conservation, National Chung-Hsing Univ., Taichung, Taiwan, ROC.

Note. Associate Editor: Keh-Han Wang. Discussion open until November 1, 2003. Separate discussions must be submitted for individual papers. To extend the closing date by one month, a written request must be filed with the ASCE Managing Editor. The manuscript for this paper was submitted for review and possible publication on December 14, 2001; approved on October 21, 2002. This paper is part of the Journal of Engineering Mechanics, Vol. 129, No. 6, June 1, 2003. CASCE, ISSN 0733-9399/2003/6-613-626/\$18.00.
}

to deal with this kind of problem; however, although their approach is convenient to use, the error cannot be explained clearly.

In the present study, new orthogonal inner products are developed to overcome the nonorthogonal eigenfunction problem. The present orthogonal inner product is the extension of the inner product of Rhodes-Robinson (1979), which dealt with capillary gravity waves in finite water depth. This concept was also adopted by Sahoo et al. (2000) to study the problem of a zero thickness floating elastic plate acted by some external forces.

\section{Potential Flow Solution}

Because there are many former studies of potential flow solution, we hence only give a brief summary.

The fluid domain of the present analysis is divided into three regions by interfaces $x= \pm b / 2$ as shown in Fig. 1. With the usual assumptions of a perfect fluid and small amplitude waves, the

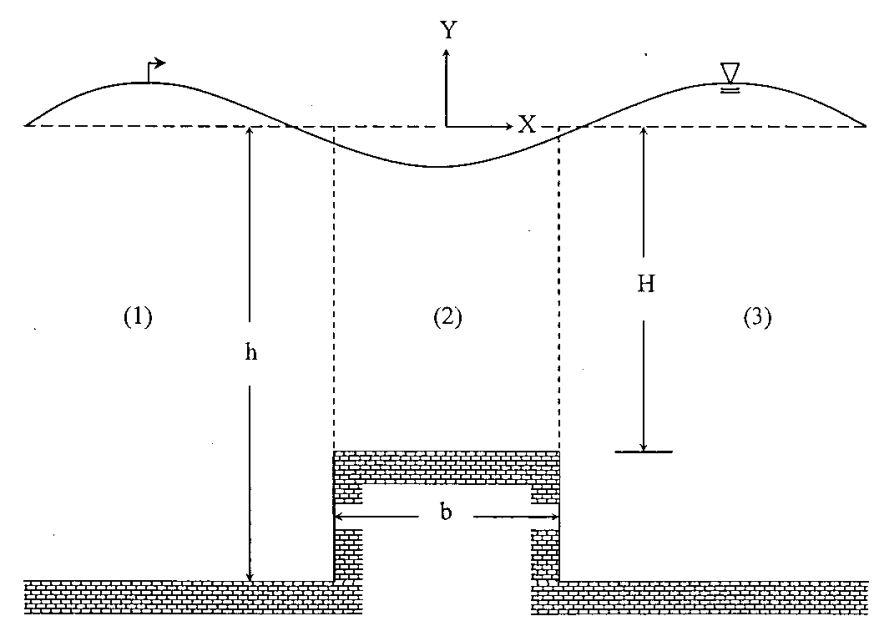

Fig. 1. Water wave passing over submerged dike 

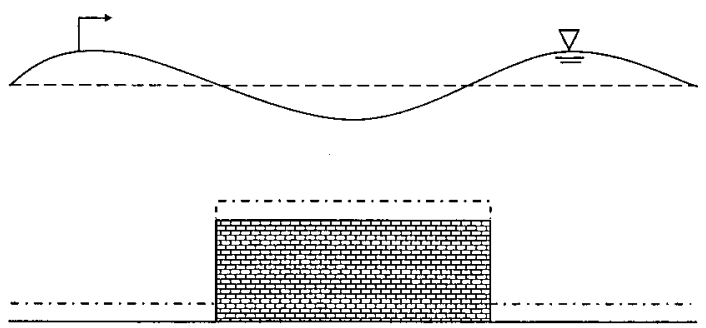

Fig. 2. Horizontal boundary layer only

velocity potential, $\Phi(x, y, t)=\phi(x, y) \cdot e^{-i \omega t}$ is necessary to satisfy Laplace's equation, the linearized free-surface condition of water wave, and the no-flux bottom boundary condition. Using the eigenfunction expansion similar to that in Ou Yang et al. (1997), we find that the spatial velocity potential $\phi^{(j)}$, after applying free surface and bottom boundary conditions, can be expressed as

$$
\begin{gathered}
\phi^{(1)}(x, y)=\frac{g \eta_{0}}{i \omega} e^{i \alpha_{0} x} f_{0}+\sum_{n=0}^{\infty} b_{n} e^{-i \alpha_{n} x} f_{n} \\
\phi^{(2)}(x, y)=\sum_{m=0}^{\infty}\left[c_{m} \cos \left(\beta_{m} x\right)+d_{m} \sin \left(\beta_{m} x\right)\right] F_{m} \\
\phi^{(3)}(x, y)=\sum_{n=0}^{\infty} e_{n} e^{i \alpha_{n} x} f_{n}
\end{gathered}
$$

where

$$
\begin{gathered}
f_{n}=\frac{\cosh \left(\alpha_{n}(y+h)\right)}{\cosh \left(\alpha_{n} h\right)} \\
F_{m}=\frac{\cosh \left(\beta_{m}(y+H)\right)}{\cosh \left(\beta_{m} H\right)}
\end{gathered}
$$

with $\eta_{0}$ being the incident wave amplitude. The propagating and evanescent wave numbers, $\alpha_{0}\left(\beta_{0}\right)$ and $\alpha_{n \geqslant l}\left(\beta_{m \geqslant l}\right)$ satisfy the usual dispersion relations

$$
\begin{gathered}
\omega^{2}=g \alpha_{n} \tanh \left(\alpha_{n} h\right), \\
\omega^{2}=g \beta_{m} \tanh \left(\beta_{m} H\right) \quad n, m=0,1,2, \ldots
\end{gathered}
$$

It can be readily shown that the wave numbers above are either real or pure imaginary, hence the corresponding eigenfunctions are orthogonal and the solution forms are complete. When boundary conditions of the continuity of normal velocity and pressure on $x= \pm b / 2$ are used, and the orthogonal conditions are applied, the coefficients $b_{n}, c_{m}, d_{m}$, and $e_{n}$ can be expressed as a matrix problem to be solved.

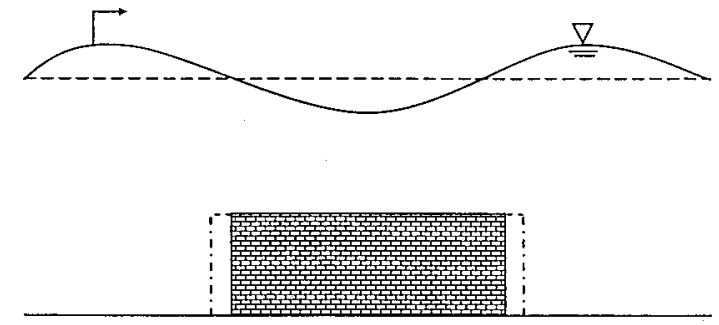

Fig. 3. Vertical boundary layer only

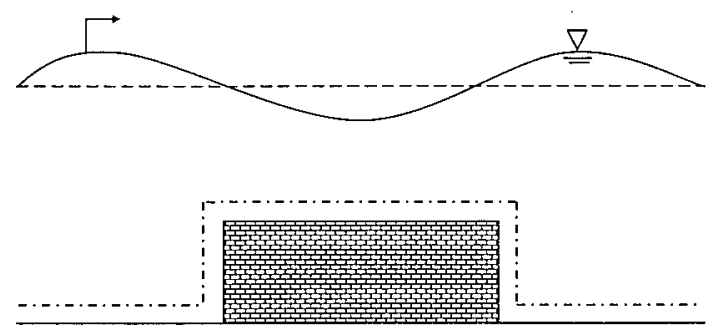

Fig. 4. Real boundary layer

\section{Viscous Flow Analysis}

\section{Governing Equations}

The fluid occupies the same regions as above. Getting rid of the time dependence factor $e^{-i \omega t}$, with $\omega$ as the angular frequency, the time-independent perturbed velocity of the water wave can be divided into an irrotational part and a rotational part by Helmholtz's theorem as follows:

$$
\mathbf{V}^{(j)}=\nabla \phi^{(j)}+\nabla \times \mathbf{U}^{(j)}
$$

Thus, the continuity equation becomes

$$
\nabla^{2} \phi^{(j)}=0
$$

and the linearized momentum equation turns into

$$
\begin{gathered}
-i \rho \omega \phi^{(j)}+P^{(j)}=0 \\
-i \rho \omega\left(\nabla \times \mathbf{U}^{(j)}\right)=\mu \nabla^{2}\left(\nabla \times \mathbf{U}^{(j)}\right)
\end{gathered}
$$

Since we consider the two-dimensional Cartesian coordinate problem, the vector potential $\mathbf{U}^{(j)}$ can be written as $\mathbf{U}^{(j)}=\varphi^{(j)} \mathbf{e}_{\mathbf{z}}$ (see Morse and Feshbach 1978, p. 1766). Therefore, Eq. (10) can be in the form

$$
\nabla^{2} \varphi^{(j)}+k_{\omega}^{2} \varphi^{(j)}=0
$$

where the constant is

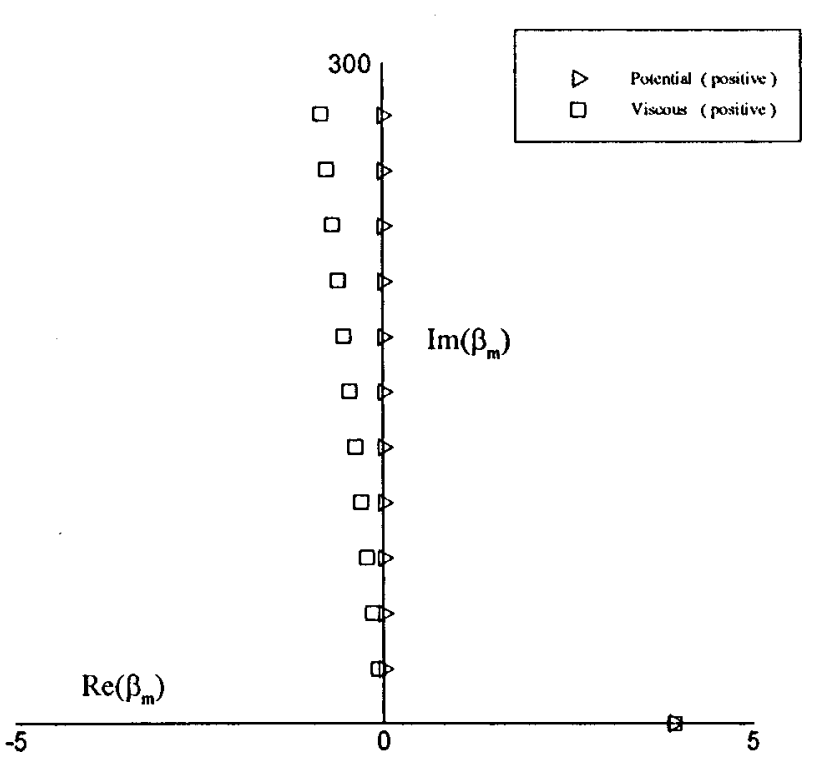

Fig. 5. Relationships of wave numbers between two models $(h / H$ $=2, b / H=2, h=0.25^{\mathrm{m}}, \eta_{0}=3.2^{\mathrm{cm}}, T=1.5^{\mathrm{s}}$ ) 


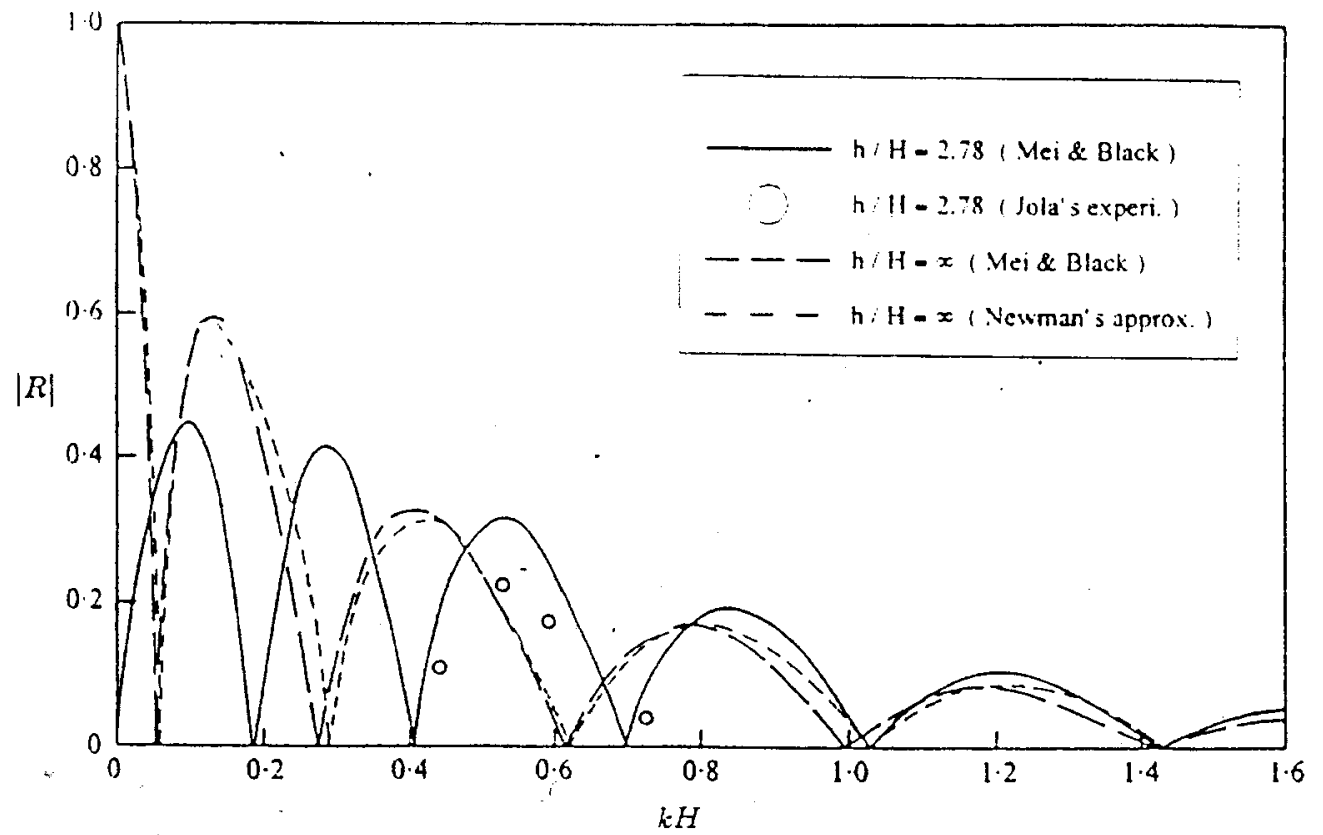

(a) Mei \& Black (1969).

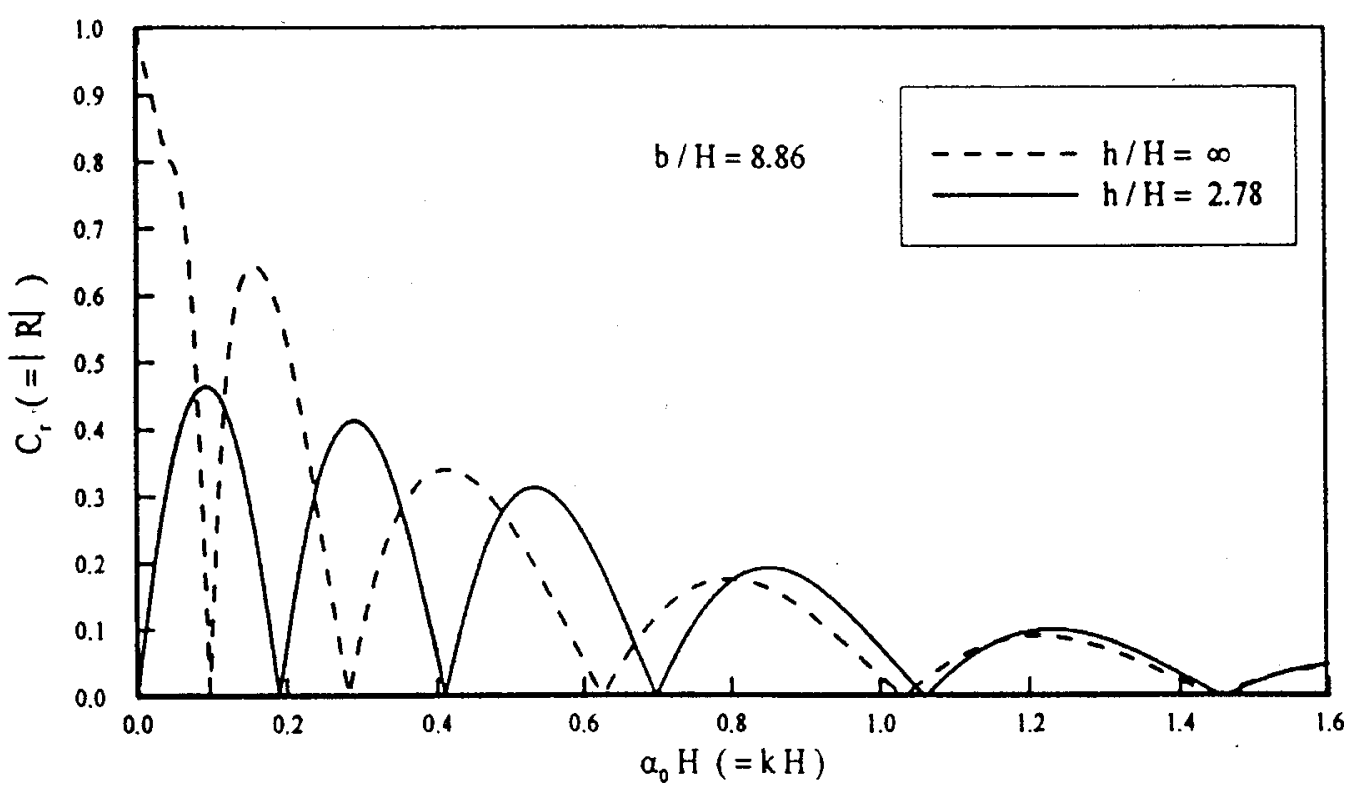

(b) Present Study.

Fig. 6. Reflection coefficient of potential model (a) Mei and Black (1969); (b) present study

$$
k_{\omega}^{2}=\frac{i \omega}{v}
$$

\section{Boundary Conditions}

The dynamic boundary conditions on the free surface, $y=0$, are the continuities of normal and tangential stresses (e.g., see Aris 1962)

$$
-i \omega \phi^{(j)}+2 v\left(\phi_{y y}^{(j)}-\varphi_{x y}^{(j)}\right)+g \eta=0
$$

$$
2 \phi_{x y}^{(j)}-\varphi_{x x}^{(j)}+\varphi_{y y}^{(j)}=0
$$

The conventional kinematic boundary condition on the free surface, $y=0$, is

$$
\phi_{y}^{(j)}-\varphi_{x}^{(j)}=-i \omega \eta
$$

Regarding the bottom boundary conditions, the no-slip condition should be satisfied, thus we get

$$
\phi_{x}^{(j)}+\varphi_{y}^{(j)}=0
$$




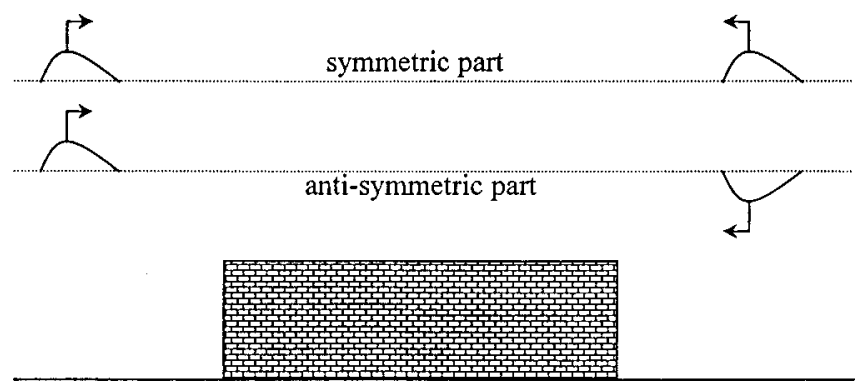

Fig. 7. Approach by Mei and Black (1969)

$$
\phi_{y}^{(j)}-\varphi_{x}^{(j)}=0
$$

for $y=-h$ at regions (1) and (3) and for $y=-H$ at region (2).

At two regional interfaces, $x= \pm b / 2$, the continuities of (a) normal fluid stress, (b) normal fluid velocity, and (c) the no-flux condition on the two vertical walls are considered. All of them are listed sequentially as follows:

At interface $x=-b / 2$

$$
\begin{gathered}
-i \omega \phi^{(1)}+2 \nu\left(\phi_{y y}^{(1)}-\varphi_{x y}^{(1)}\right)=-i \omega \phi^{(2)}+2 \nu\left(\phi_{y y}^{(2)}-\varphi_{x y}^{(2)}\right) \\
-H \leqslant y \leqslant 0 \\
\phi_{x}^{(1)}+\varphi_{y}^{(1)}=\phi_{x}^{(2)}+\varphi_{y}^{(2)}, \quad-H \leqslant y \leqslant 0 \\
\phi_{x}^{(1)}+\varphi_{y}^{(1)}=0, \quad-h \leqslant y \leqslant-H
\end{gathered}
$$

At interface $x=b / 2$

$$
\begin{gathered}
-i \omega \phi^{(3)}+2 \nu\left(\phi_{y y}^{(3)}-\varphi_{x y}^{(3)}\right)=-i \omega \phi^{(2)}+2 \nu\left(\phi_{y y}^{(2)}-\varphi_{x y}^{(2)}\right) \\
-H \leqslant y \leqslant 0 \\
\phi_{x}^{(3)}+\varphi_{y}^{(3)}=\phi_{x}^{(2)}+\varphi_{y}^{(2)}, \quad-H \leqslant y \leqslant 0
\end{gathered}
$$

$$
\phi_{x}^{(3)}+\varphi_{y}^{(3)}=0, \quad-h \leqslant y \leqslant-H
$$

\section{Method of Solution}

\section{Solution Forms}

To solve Eqs. (8) and (11) with the conditions (14), (16), and (17), once again, the eigenfunction expansion method is used. These spatial potentials in each region can be found as

$$
\phi^{(1)}(x, y)=A_{0} e^{i \alpha_{0} x}\left[\cosh \left(\alpha_{0} y\right)+P_{0} \sinh \left(\alpha_{0} y\right)\right]
$$$$
+\sum_{n=0}^{\infty} B_{n} e^{-i \alpha_{n} x}\left[\cosh \left(\alpha_{n} y\right)+P_{n} \sinh \left(\alpha_{n} y\right)\right]
$$

$$
\varphi^{(1)}(x, y)=A_{0} e^{i \alpha_{0} x}\left[Q_{0} e^{\hat{\alpha}_{0}(y+h)}+R_{0} e^{-\hat{\alpha}_{0}(y+h)}\right]
$$$$
+\sum_{n=0}^{\infty} B_{n} e^{-i \alpha_{n} x}\left[Q_{n} e^{\hat{\alpha}_{n}(y+h)}+R_{n} e^{-\hat{\alpha}_{n}(y+h)}\right]
$$

$$
\begin{aligned}
\phi^{(2)}(x, y)= & \sum_{m=0}^{\infty}\left(C_{m} \cos \left(\beta_{m} x\right)+D_{m} \sin \left(\beta_{m} x\right)\right) \\
& \times\left[\cosh \left(\beta_{m} y\right)+\widetilde{P}_{m} \sinh \left(\beta_{m} y\right)\right] \\
\varphi^{(2)}(x, y)= & \sum_{m=0}^{\infty}\left(C_{m} \cos \left(\beta_{m} x\right)+D_{m} \sin \left(\beta_{m} x\right)\right)
\end{aligned}
$$

$$
\times\left[\widetilde{Q}_{m} e^{\hat{\beta}_{m}(y+H)}+\widetilde{R}_{m} e^{-\hat{\beta}_{m}(y+H)}\right]
$$

$$
\phi^{(3)}(x, y)=\sum_{n=0}^{\infty} E_{n} e^{i \alpha_{n} x}\left[\cosh \left(\alpha_{n} y\right)+P_{n} \sinh \left(\alpha_{n} y\right)\right]
$$

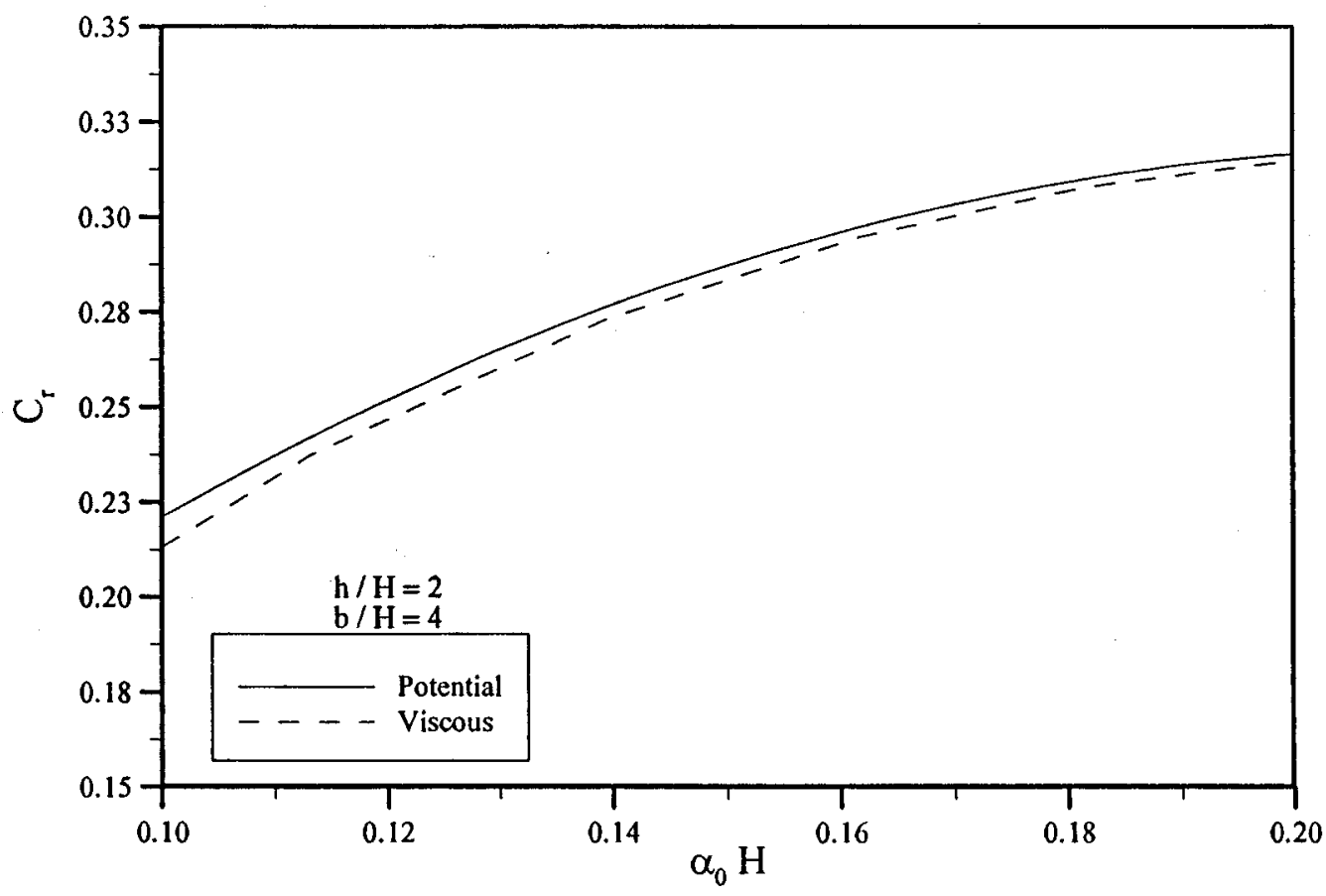

Fig. 8. Difference of reflection coefficient between two models $\left(\eta_{0}=3.2^{\mathrm{cm}}, T=1.5^{\mathrm{s}}, \quad v=1.17 \times 10^{-6^{\mathrm{m}^{2} / \mathrm{s}}}\right)$ 

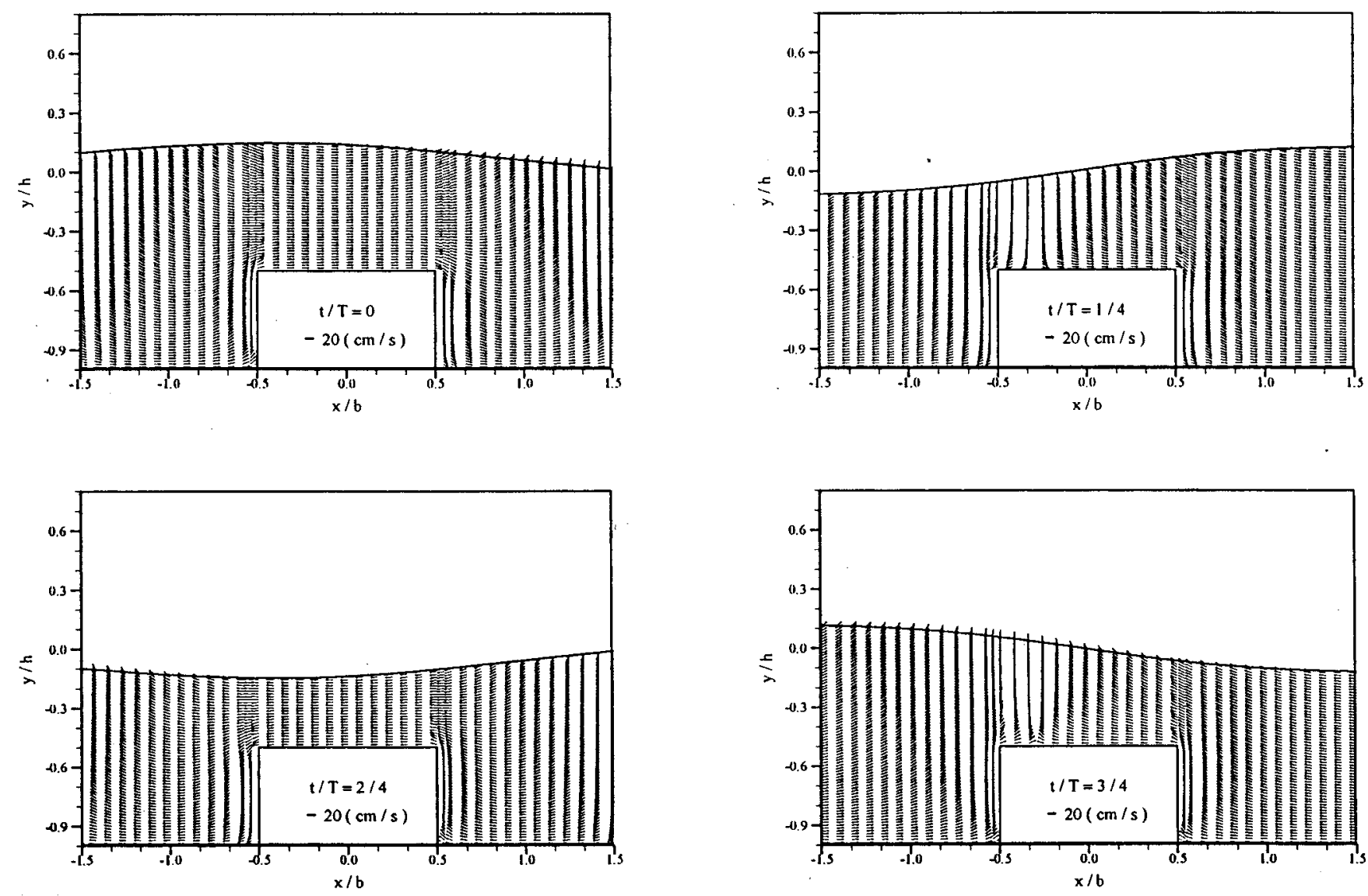

Fig. 9. Surface elevation and velocity field of potential model $\left(h / H=2, b / H=2, h=0.25^{\mathrm{m}}, \eta_{0}=3.2^{\mathrm{cm}}, T=1.5^{\mathrm{s}}\right)$

$$
\varphi^{(3)}(x, y)=\sum_{n=0}^{\infty} E_{n} e^{i \alpha_{n} x}\left[Q_{n} e^{\hat{\alpha}_{n}(y+h)}+R_{n} e^{-\hat{\alpha}_{n}(y+h)}\right]
$$

where

$$
\begin{aligned}
& \hat{\alpha}_{n}^{2}=\alpha_{n}^{2}-k_{\omega}^{2}, \operatorname{Re}\left(\hat{\alpha}_{n}\right) \leqslant 0 \\
& P_{n}=\mathrm{F}^{2}\left\{\Delta_{n}\left[2 \Omega_{n}^{2} \cosh \Omega_{n}-\Delta_{n} \cosh \Theta_{n}\right]\right\} /\left\{\Omega_{n} S_{n}\right\}
\end{aligned}
$$

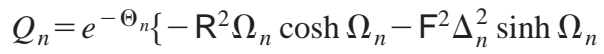

$$
\begin{aligned}
& \left.+2 \mathrm{~F}^{2} \Omega_{n} \Theta_{n}\left[e^{\Theta_{n}} \Delta_{n}-2 \Omega_{n}^{2} \cosh \Omega_{n}\right]\right\} /\left\{-2 i \Theta n S_{n}\right\}
\end{aligned}
$$

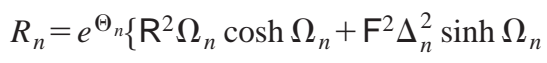

$$
\begin{aligned}
& \left.+2 \mathrm{~F}^{2} \Omega_{n} \Theta_{n}\left[\Delta_{n}-2 \Omega_{n}^{2} \cosh \Omega_{n} e^{\Theta_{n}}\right]\right\} /\left\{-2 i \Theta_{n} S_{n}\right\}
\end{aligned}
$$

$$
\begin{gathered}
S_{n}=\mathrm{R}^{2} \cosh \Theta_{n}+2 \mathrm{~F}^{2} \Omega_{n} \Delta_{n} \sinh \Omega_{n}-4 \mathrm{~F}^{2} \Omega_{n}^{2} \Theta_{n} \sinh \Theta_{n} \\
\mathrm{R}=\frac{\omega h^{2}}{v} \\
\mathrm{~F}=\frac{\omega h}{\sqrt{g h}} \\
\Omega_{n}=\alpha_{n} h \\
\Theta_{n}=\hat{\alpha}_{n} h \\
\Delta_{n}=2 \Omega_{n}^{2}-i \mathrm{R}
\end{gathered}
$$

and wave numbers $\alpha_{n}$, solved by the combination of Eqs. (13) and (15), are the roots of the following equations:

$$
\begin{aligned}
& \mathrm{F}^{2} \cosh \left(\alpha_{n} h\right)-\left(\alpha_{n} h\right) \sinh \left(\alpha_{n} h\right)+\left(\alpha_{n} h\right)\left[\left(\alpha_{n} h\right) \cosh \left(\alpha_{n} h\right)-\mathrm{F}^{2} \sinh \left(\alpha_{n} h\right)\right] \frac{\tanh \left(\hat{\alpha}_{n} h\right)}{\hat{\alpha}_{n} h}+4 i \frac{\mathrm{F}^{2}}{\mathrm{R}}\left(\alpha_{n} h\right)^{2} \cosh \left(\alpha_{n} h\right) \\
& \quad-8 i \frac{\mathrm{F}^{2}}{\mathrm{R}}\left(\alpha_{n} h\right)^{3} \sinh \left(\alpha_{n} h\right) \frac{\tanh \left(\hat{\alpha}_{n} h\right)}{\hat{\alpha}_{n} h}-8 \frac{\mathrm{F}^{2}}{\mathrm{R}^{2}}\left(\alpha_{n} h\right)^{4} \cosh \left(\alpha_{n} h\right)+8 \frac{\mathrm{F}^{2}}{\mathrm{R}^{2}}\left(\alpha_{n} h\right)^{5} \sinh \left(\alpha_{n} h\right) \frac{\tanh \left(\hat{\alpha}_{n} h\right)}{\hat{\alpha}_{n} h}-4 i \frac{\mathrm{F}^{2}}{\mathrm{R}}\left(\alpha_{n} h\right)^{2} \frac{1}{\cosh \left(\hat{\alpha}_{n} h\right)} \\
& \quad+8 \frac{\mathrm{F}^{2}}{\mathrm{R}^{2}}\left(\alpha_{n} h\right)^{4} \frac{1}{\cosh \left(\hat{\alpha}_{n} h\right)}=0
\end{aligned}
$$



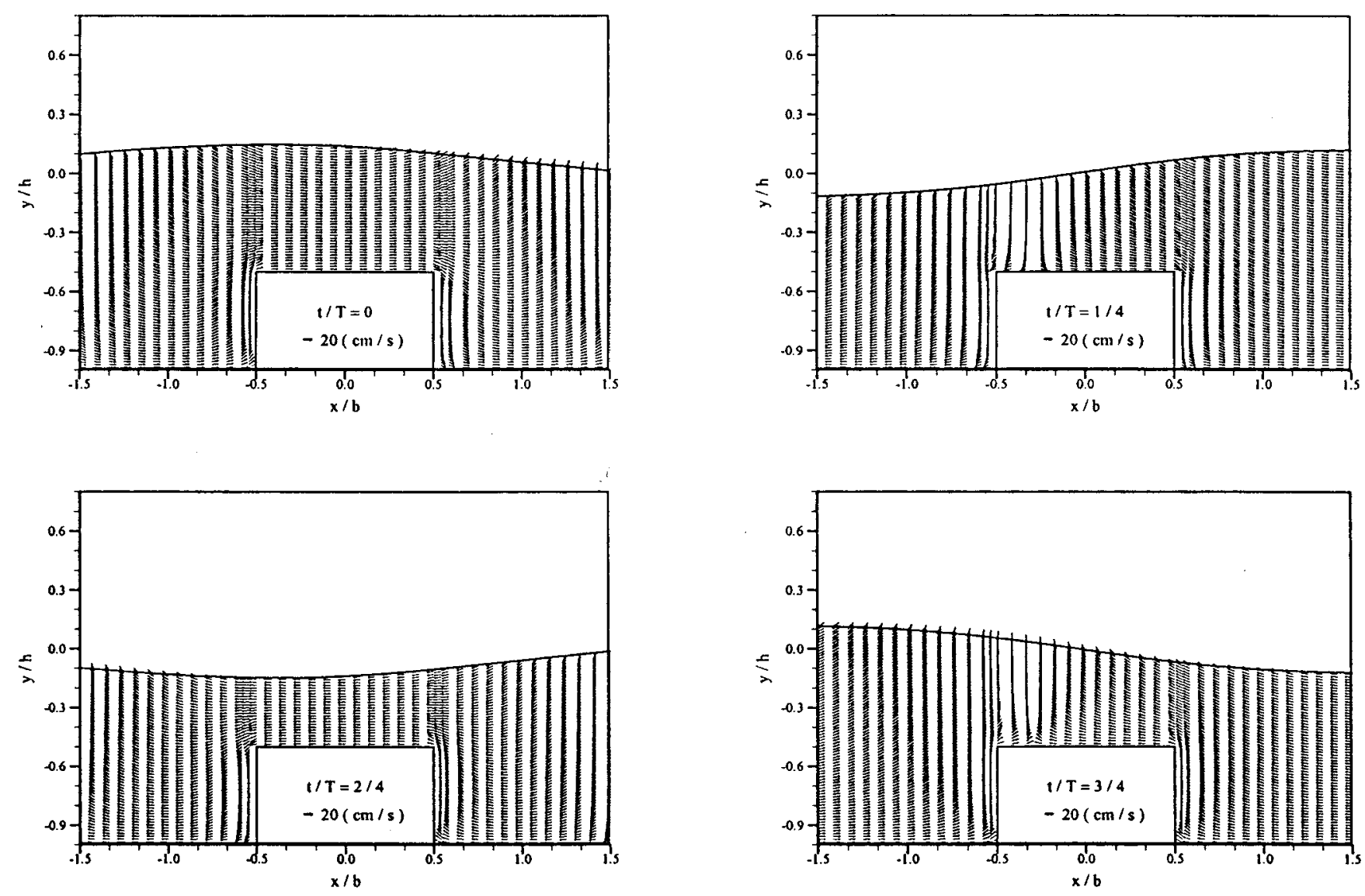

Fig. 10. Surface elevation and velocity field of viscous model $\left(h / H=2, \quad b / H=2, h=0.25^{\mathrm{m}}, \quad \eta_{0}=3.2^{\mathrm{cm}}, \quad T=1.5^{\mathrm{s}}, \quad v=1.17 \times 10^{-6^{\mathrm{m}^{2} / \mathrm{s}}}\right)$

For the shallow region, region (2), the quantity $\beta_{m}, \hat{\beta}_{m}, \widetilde{P}_{m}$, $\widetilde{Q}_{m}$, and $\widetilde{R}_{m}$ are defined similarly by replacing $h$ and $\alpha_{n}$ with $H$ and $\beta_{m}$ in Eqs. (30) $-(40)$.

Paying attention to the terms involving the coefficient $A_{0}$ appear in the first term of the right hand side of Eqs. (24) and (25), they actually stand for the given incident wave. The incident wave can be solved by Eqs. (8) and (11) with boundary conditions (13)-(17). $A_{0}$ can be obtained as

$$
A_{0}=\frac{\omega h \eta_{0}}{i \mathrm{~F}^{2}} \frac{\mathrm{R}}{\mathrm{R}+2 i \Omega_{0}^{2}+2 \Omega_{0} \Theta_{0}\left[e^{\Theta_{0}} Q_{0}-e^{-\Theta_{0} R_{0}}\right]}
$$

by substituting the solution form [the first term of the right hand side of Eqs. (24) and (25)] into Eq. (15). The constant $\eta_{0}$ is the incident wave amplitude at $x=0$ and the propagating wave number $\alpha_{0}$ is one of the roots of Eq. (40) whose real part is dominated.

The unknown coefficients $B_{n}, C_{m}, D_{m}$, and $E_{n}$ now will be solved by interface conditions (18)-(23). One can notice that the orthogonality of the eigenfunctions no longer exists because of wave numbers being complex.

\section{Orthogonal Inner Products}

To investigate the surface tension effect of water waves, RhodesRobinson (1979) rewrote the governing ordinary differential equation and its corresponding boundary conditions, in water depth direction, as the form of a typical Sturm-Liouville problem with modification then developed a so-called orthogonal inner product to overcome the predicament resulting from the nonorthogonality of the eigenfunctions.
Referring to Rhodes-Robinson's treatment, the sets of functions, which express the dependence on depth in the separated functions in region (1) and (3), can be written as the following boundary-value problems. Governing Eq. (8) with boundary conditions (17) and the combination of Eqs. (13) and (15) gives

$$
\begin{gathered}
Y_{n}^{\prime \prime}-\alpha_{n}^{2} Y_{n}=0 \\
Y_{n}^{\prime}(-h)=i \alpha_{n} \hat{Y}_{n}(-h) \\
\omega\left(-i \omega+2 v \alpha_{n}^{2}\right) Y_{n}(0)+i g Y_{n}^{\prime}(0) \\
=-g \alpha_{n} \hat{Y}_{n}(0)+2 i v \omega \alpha_{n} \hat{Y}_{n}^{\prime}(0)
\end{gathered}
$$

While governing Eq. (11) with boundary conditions (16) and (14) gives

$$
\begin{gathered}
\hat{Y}_{n}^{\prime \prime}-\left(\alpha_{n}^{2}-k_{\omega}^{2}\right) \hat{Y}_{n}=0 \\
\hat{Y}_{n}^{\prime}(-h)=-i \alpha_{n} Y_{n}(-h) \\
\left(k_{\omega}^{2}-2 \alpha_{n}^{2}\right) \hat{Y}_{n}(0)=2 i \alpha_{n} Y_{n}^{\prime}(0)
\end{gathered}
$$

where

$$
Y_{n}=\cosh \left(\alpha_{n} y\right)+P_{n} \sinh \left(\alpha_{n} y\right)
$$

stand for the irrotational parts, and

$$
\hat{Y}_{n}=Q_{n} e^{\hat{\alpha}_{n}(y+h)}+R_{n} e^{-\hat{\alpha}_{n}(y+h)}
$$

are the solutions of rotational potential. From the above modified Sturm-Liouville problems, Eqs. (42)-(44) and (45)-(47), we can define the orthogonal inner product of irrotational potentials for region (1) and, similarly, for region (3) as 


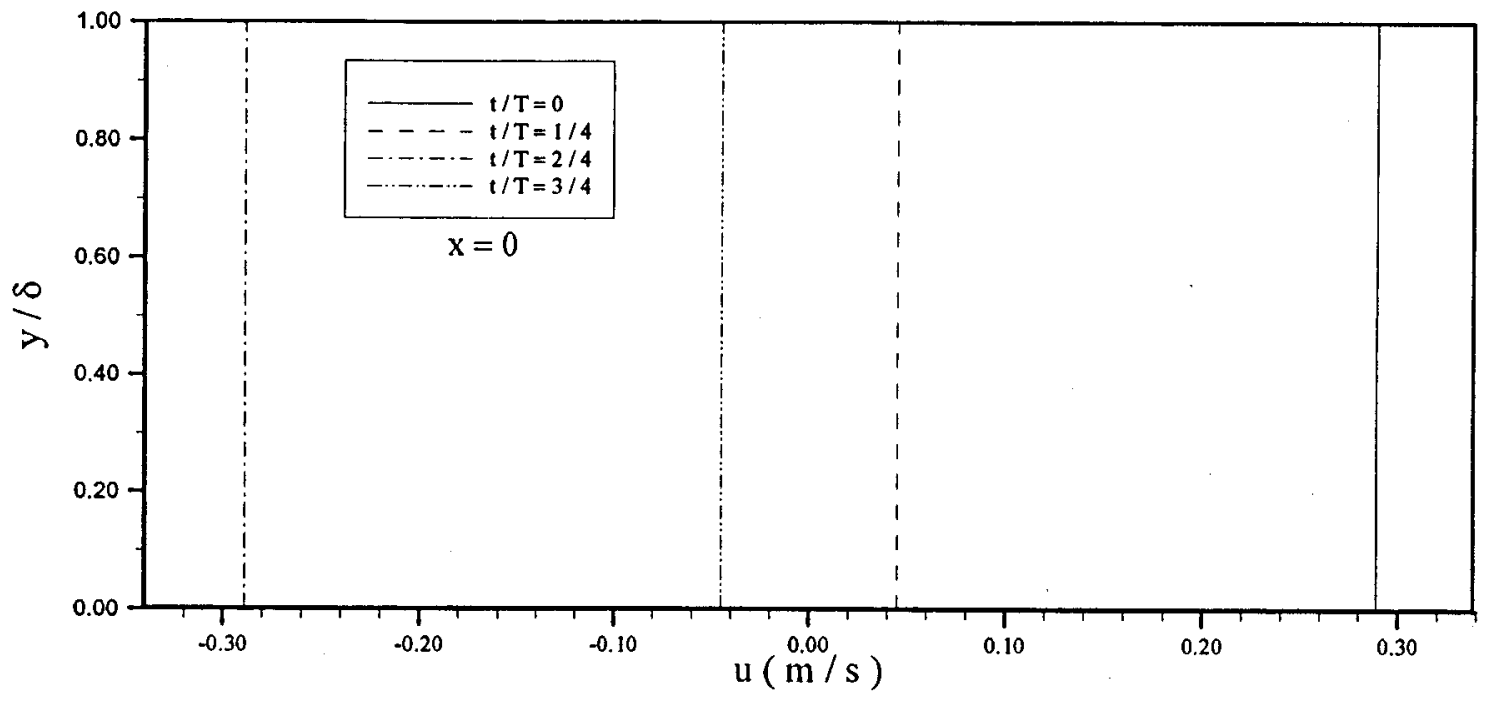

Fig. 11. Velocity profile within presumed boundary layer of potential model $\left(h / H=2, b / H=2, h=0.25^{\mathrm{m}}, \quad \eta_{0}=3.2^{\mathrm{cm}}, T=1.5^{\mathrm{s}}\right)$

$\left\langle Y_{m}, Y_{n}\right\rangle$

$$
\left\{\begin{array}{l}
\int_{-h}^{0} Y_{m}(y) Y_{n}(y) d y, \quad m=n \\
\int_{-h}^{0} Y_{m}(y) Y_{n}(y) d y+\frac{4 v}{g}(-i \omega) \\
-\frac{\alpha_{n}^{2} \hat{\alpha}_{m}-\alpha_{m}^{2} \hat{\alpha}_{n}}{\left(\alpha_{m} \alpha_{n}\right)^{2}\left(\alpha_{m}^{2}-\alpha_{n}^{2}\right)} Y_{m}^{\prime}(-h) Y_{n}^{\prime}(-h) \\
+\frac{2 i}{\alpha_{m}^{2}-\alpha_{n}^{2}}\left\{\frac{\hat{\alpha}_{m}}{\alpha_{m}} R_{m} Y_{n}^{\prime}(-h)-\frac{\hat{\alpha}_{n}}{\alpha_{n}} R_{n} Y_{m}^{\prime}(-h)\right\}, \quad m \neq n
\end{array}\right.
$$

Of course, by the same procedures we can obtain the orthogonal inner product of irrotational potential for region (2) as

$$
\left\langle\widetilde{Y}_{m}, \widetilde{Y}_{n}\right\rangle
$$

$$
\left\{\begin{array}{l}
\int_{-H}^{0} \widetilde{Y}_{m}(y) \widetilde{Y}_{n}(y) d y, \quad m=n \\
\int_{-H}^{0} \widetilde{Y}_{m}(y) \widetilde{Y}_{n}(y) d y+\frac{4 v}{g}(-i \omega) \\
-\frac{\beta_{n}^{2} \hat{\beta}_{m}-\beta_{m}^{2} \hat{\beta}_{n}}{\left(\beta_{m} \beta_{n}\right)^{2}\left(\beta_{m}^{2}-\beta_{n}^{2}\right)} \widetilde{Y}_{m}^{\prime}(-H) \widetilde{Y}_{n}^{\prime}(-H) \\
+\frac{2 i}{\beta_{m}^{2}-\beta_{n}^{2}}\left\{\frac{\hat{\beta}_{m}}{\beta_{m}} \widetilde{R}_{m} \widetilde{Y}_{n}^{\prime}(-H)-\frac{\hat{\beta}_{n}}{\beta_{n}} \widetilde{R}_{n} \widetilde{Y}_{m}^{\prime}(-H)\right\}, \quad m \neq n
\end{array}\right.
$$

with

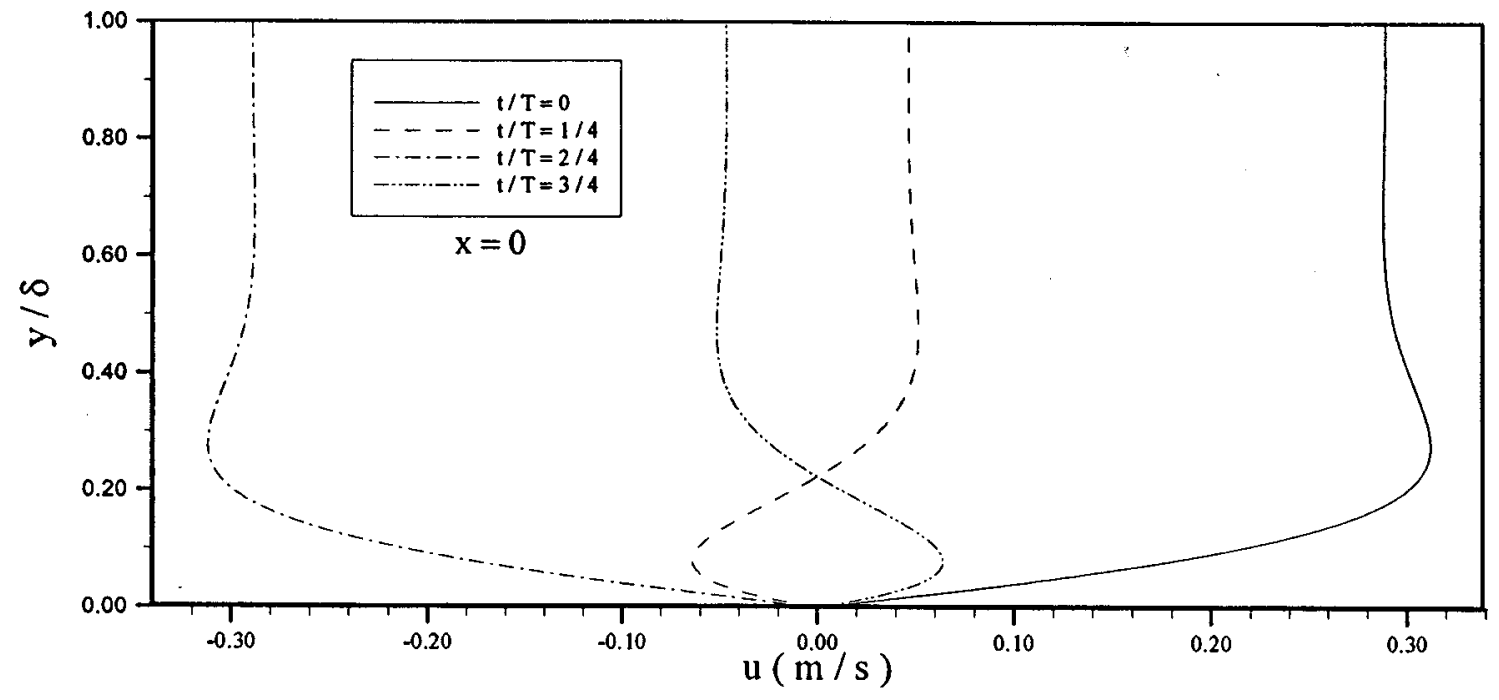

Fig. 12. Velocity profile within boundary layer of viscous model $\left(h / H=2, b / H=2, h=0.25^{\mathrm{m}}, \quad \eta_{0}=3.2^{\mathrm{cm}}, \quad T=1.5^{\mathrm{s}}, \nu=1.17 \times 10^{-6^{\mathrm{m}^{2} / \mathrm{s}}}\right)$ 

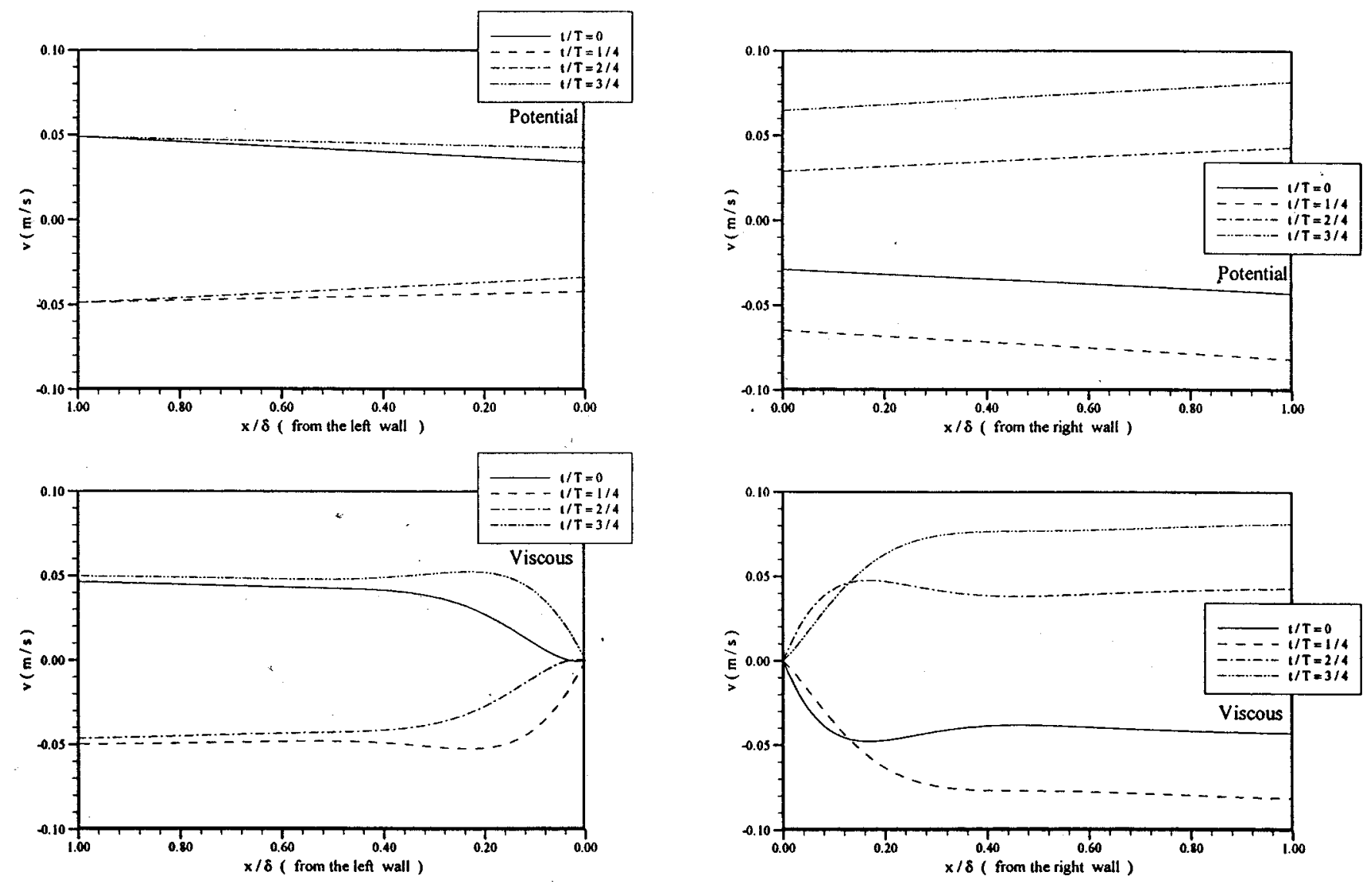

Fig. 13. Velocity profile at midpoint of vertical wall within boundary layer $\left(h / H=2, b / H=2, h=0.25^{\mathrm{m}}, \eta_{0}=3.2^{\mathrm{cm}}, T=1.5^{\mathrm{s}}, \quad v=1.17\right.$ $\times 10^{-6^{\mathrm{m}^{2} / \mathrm{s}}}$ )

$$
\widetilde{Y}_{m}=\cosh \left(\beta_{m} y\right)+\widetilde{P}_{m} \sinh \left(\beta_{m} y\right)
$$

Then, it is easy to find that for $m \neq n$

$$
\begin{aligned}
& \left\langle Y_{m}, Y_{n}\right\rangle=0 \\
& \left\langle\widetilde{Y}_{m}, \widetilde{Y}_{n}\right\rangle=0
\end{aligned}
$$

which indicate that the set of functions $\left\{Y_{n} ; n=0,1,2, \ldots\right\}$ and $\left\{\widetilde{Y}_{m} ; m=0,1,2, \ldots\right\}$ appear in Eqs. (24)-(29), are orthogonal with respect to the inner product defined by Eqs. (50) and (51). Eqs. (50) and (51) can also be written as

$$
\begin{aligned}
& \int_{-h}^{0} Y_{m}(y) Y_{n}(y) d y \\
& \quad=\left\{\begin{array}{l}
\left\langle Y_{m}, Y_{n}\right\rangle, \quad m=n \\
-\frac{4 v}{g}(-i \omega) \\
+\frac{\alpha_{n}^{2} \hat{\alpha}_{m}-\alpha_{m}^{2} \hat{\alpha}_{n}}{\left(\alpha_{m} \alpha_{n}\right)^{2}\left(\alpha_{m}^{2}-\alpha_{n}^{2}\right)} Y_{m}^{\prime}(-h) Y_{n}^{\prime}(-h) \\
-\frac{2 i}{\alpha_{m}^{2}-\alpha_{n}^{2}}\left\{\frac{\hat{\alpha}_{m}}{\alpha_{m}} R_{m} Y_{n}^{\prime}(-h)-\frac{\hat{\alpha}_{n}}{\alpha_{n}} R_{n} Y_{m}^{\prime}(-h)\right\}, \quad m \neq n
\end{array}\right.
\end{aligned}
$$

$$
\begin{aligned}
& \int_{-h}^{0} \widetilde{Y}_{m}(y) \widetilde{Y}_{n}(y) d y \\
& =\left\{\begin{array}{l}
\left\langle\widetilde{Y}_{m}, \widetilde{Y}_{n}\right\rangle, \quad m=n \\
-\frac{4 v}{g}(-i \omega) \\
+\frac{\beta_{n}^{2} \hat{\beta}_{m}-\beta_{m}^{2} \hat{\beta}_{n}}{\left(\beta_{m} \beta_{n}\right)^{2}\left(\beta_{m}^{2}-\beta_{n}^{2}\right)} \widetilde{Y}_{m}^{\prime}(-H) \widetilde{Y}_{n}^{\prime}(-H) \\
-\frac{2 i}{\beta_{m}^{2}-\beta_{n}^{2}}\left\{\frac{\hat{\beta}_{m}}{\beta_{m}} \widetilde{R}_{m} \widetilde{Y}_{n}^{\prime}(-H)-\frac{\hat{\beta}_{n}}{\beta_{n}} \widetilde{R}_{n} \widetilde{Y}_{m}^{\prime}(-H)\right\}, \quad m \neq n
\end{array}\right.
\end{aligned}
$$

From Eqs. $\left(50^{\prime}\right)$ and $\left(51^{\prime}\right)$, we find that we need to perform integration only when $m=n$, while when $m \neq n$, although it is not zero as the minimal squares method usually assumed, the integration is still easy to obtain by applying the present orthogonal inner product method. By looking into the inner products above, we can realize that terms beside the integrals in Eqs. (50) and (51) are errors of the minimal squares method applied by Lo and Lee (2000). This indicates that the present orthogonal inner product approach can reduce the error of the minimal squares method.

At interface $x=-b / 2$, applying Eqs. (51') to Eq. (18) and Eq. $\left(50^{\prime}\right)$ to Eqs. (19) and (20), we have 

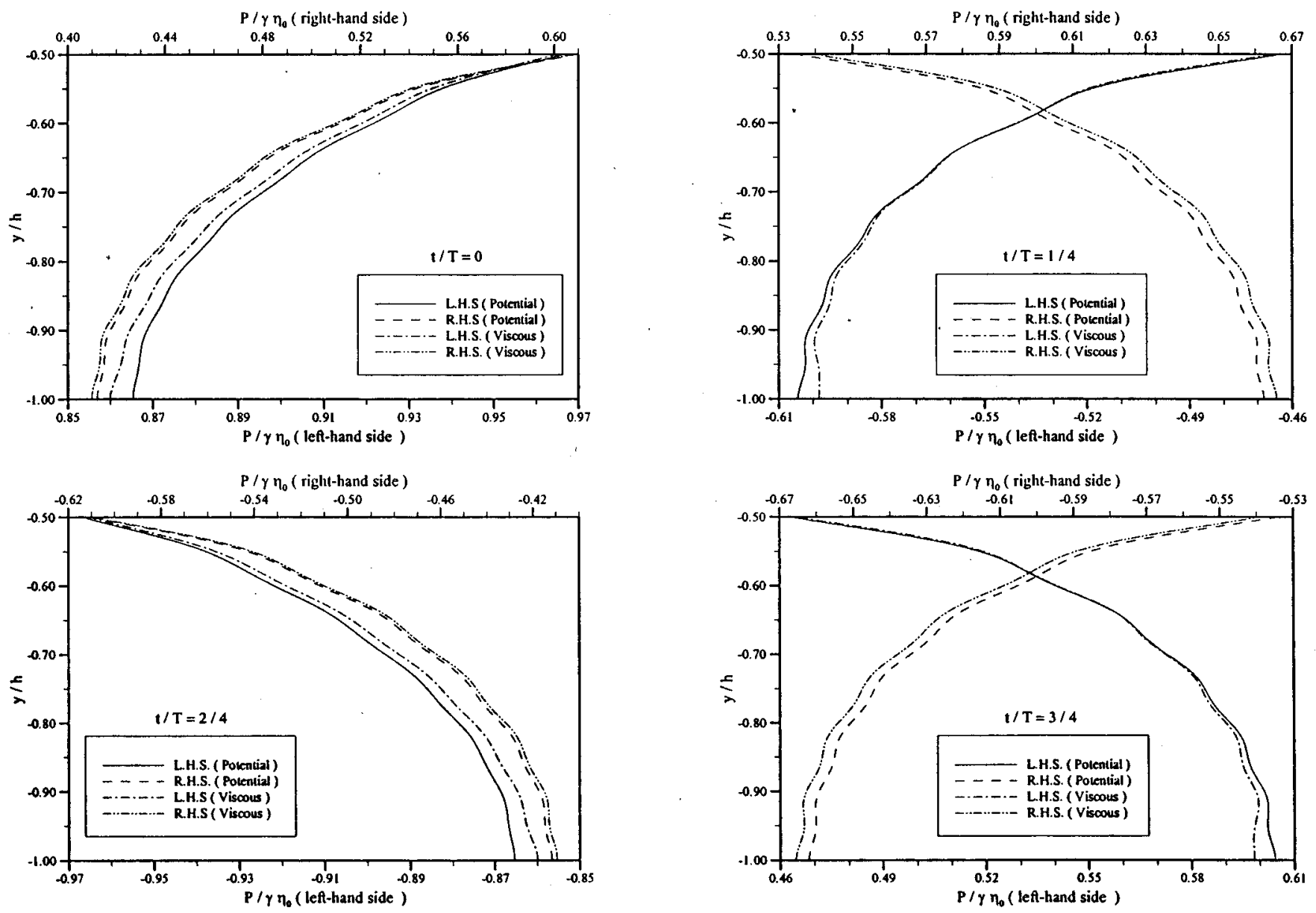

Fig. 14. Pressure distribution on vertical wall $\left(h / H=2, \quad b / H=2, h=0.25^{\mathrm{m}}, \quad \eta_{0}=3.2^{\mathrm{cm}}, \quad T=1.5^{\mathrm{s}}, \quad v=1.17 \times 10^{-6^{\mathrm{m}^{2} / \mathrm{s}}}\right)$

$$
\begin{aligned}
& \sum_{n=0}^{\infty}\left\{\left(1+2 i \frac{v}{\omega} \alpha_{n}^{2}\right) \cdot \widetilde{\Gamma}_{n \hat{m}}-2 \frac{v}{\omega} \alpha_{n} \hat{\alpha}_{n} \cdot \widetilde{\Lambda}_{n \hat{m}}\right\} e^{i \alpha_{n} \cdot b / 2} B_{n} \\
& +\sum_{m=0}^{\infty}\left\{-\left(1+2 i \frac{\nu}{\omega} \beta_{m}^{2}\right) \cdot \int_{-H}^{0} \tilde{Y}_{m} \tilde{Y}_{\hat{m}} d y+2 i \frac{\nu}{\omega} \beta_{m} \hat{\beta}_{m} \tan \left(b / 2 \cdot \beta_{m}\right) \cdot \tilde{\Pi}_{m \hat{m}}\right\} \cos \left(b / 2 \cdot \beta_{m}\right) C_{m} \\
& +\sum_{m=0}^{\infty}\left\{-\left(1+2 i \frac{v}{\omega} \beta_{m}^{2}\right) \cdot \int_{-H}^{0} \widetilde{Y}_{m} \widetilde{Y}_{\hat{m}} d y-2 i \frac{v}{\omega} \beta_{m} \hat{\beta}_{m} \cot \left(b / 2 \cdot \beta_{m}\right) \cdot \tilde{\Pi}_{m \hat{m}}\right\} \sin \left(-b / 2 \cdot \beta_{m}\right) D_{m} \\
& =\left\{-\left(1+2 i \frac{\nu}{\omega} \alpha_{0}^{2}\right) \cdot \tilde{\Gamma}_{0 \hat{m}}-2 \frac{\nu}{\omega} \alpha_{0} \hat{\alpha}_{0} \cdot \tilde{\Lambda}_{0 \hat{m}}\right\} e^{-i \alpha_{0} \cdot b / 2} A_{0}, \quad \hat{m}=0,1,2, \ldots \\
& \sum_{n=0}^{\infty}\left\{-i \alpha_{n} \cdot \int_{-h}^{0} Y_{n} Y_{\hat{n}} d y+\hat{\alpha}_{n} \cdot \hat{\Lambda}_{n \hat{n}}\right\} e^{i \alpha_{n} \cdot b / 2} B_{n}+\sum_{m=0}^{\infty}\left\{\beta_{m} \tan \left(\beta_{m} x\right) \cdot \hat{\Gamma}_{m \hat{n}}-\hat{\beta}_{m} \cdot \hat{\Pi}_{m \hat{n}}\right\} \cos \left(b / 2 \cdot \beta_{m}\right) C_{m} \\
& +\sum_{m=0}^{\infty}\left\{-\beta_{m} \cot \left(\beta_{m} x\right) \cdot \hat{\Gamma}_{m \hat{n}}-\hat{\beta}_{m} \cdot \hat{\Pi}_{m \hat{n}}\right\} \sin \left(-b / 2 \cdot \beta_{m}\right) D_{m} \\
& =\left\{-i \alpha_{0} \cdot \int_{-h}^{0} Y_{0} Y_{\hat{n}} d y-\hat{\alpha}_{0} \cdot \hat{\Lambda}_{0 \hat{n}}\right\} e^{-i \alpha_{0} \cdot b / 2} A_{0}, \quad \hat{n}=0,1,2, \ldots
\end{aligned}
$$

At interface $x=b / 2$, applying Eq. $\left(51^{\prime}\right)$ to Eq. (21) and Eq. $\left(50^{\prime}\right)$ to Eqs. (22) and (23), we have 


$$
\begin{aligned}
& \sum_{m=0}^{\infty}\left\{-\left(1+2 i \frac{v}{\omega} \beta_{m}^{2}\right) \cdot \int_{-H}^{0} \widetilde{Y}_{m} \widetilde{Y}_{\hat{m}} d y-2 i \frac{\nu}{\omega} \beta_{m} \hat{\beta}_{m} \tan \left(b / 2 \cdot \beta_{m}\right) \cdot \widetilde{\Pi}_{m \hat{m}}\right\} \cos \left(b / 2 \cdot \beta_{m}\right) C_{m} \\
& \quad+\sum_{m=0}^{\infty}\left\{-\left(1+2 i \frac{v}{\omega} \beta_{m}^{2}\right) \cdot \int_{-H}^{0} \widetilde{Y}_{m} \widetilde{Y}_{\hat{m}} d y+2 i \frac{v}{\omega} \beta_{m} \hat{\beta}_{m} \cot \left(b / 2 \cdot \beta_{m}\right) \cdot \widetilde{\Pi}_{m \hat{m}}\right\} \sin \left(b / 2 \cdot \beta_{m}\right) D_{m} \\
& \quad+\sum_{n=0}^{\infty}\left\{\left(1+2 i \frac{v}{\omega} \alpha_{n}^{2}\right) \cdot \widetilde{\Gamma}_{n \hat{m}}+2 \frac{v}{\omega} \alpha_{n} \hat{\alpha}_{n} \cdot \widetilde{\Lambda}_{n \hat{m}}\right\} e^{i \alpha_{n} \cdot b / 2} E_{n}=0, \quad \hat{m}=0,1,2, \ldots
\end{aligned}
$$

$$
\begin{aligned}
& \sum_{m=0}^{\infty}\{\left.-\beta_{m} \tan \left(b / 2 \cdot \beta_{m}\right) \cdot \hat{\Gamma}_{m \hat{n}}-\hat{\beta}_{m} \cdot \hat{\Pi}_{m \hat{n}}\right\} \cos \left(b / 2 \cdot \beta_{m}\right) C_{m} \\
&+\sum_{m=0}^{\infty}\left\{-\beta_{m} \cot \left(b / 2 \cdot \beta_{m}\right) \cdot \hat{\Gamma}_{m \hat{n}}+\hat{\beta}_{m} \cdot \hat{\Pi}_{m \hat{n}}\right\} \sin \left(b / 2 \cdot \beta_{m}\right) D_{m} \\
&-\sum_{n=0}^{\infty}\left\{-i \alpha_{n} \cdot \int_{-h}^{0} Y_{n} Y_{\hat{n}} d y-\hat{\alpha}_{n} \cdot \hat{\Lambda}_{n \hat{n}}\right\} e^{i \alpha_{n} \cdot b / 2} E_{n}=0 \\
& \hat{n}=0,1,2, \ldots
\end{aligned}
$$

where

$$
\begin{aligned}
& \widetilde{\Gamma}_{n m}=\int_{-H}^{0} Y_{n} \widetilde{Y}_{m} d y \\
& \tilde{\Lambda}_{n m}=\int_{-H}^{0} \frac{\hat{Y}_{n}^{\prime} \tilde{Y}_{m}}{\hat{\alpha}_{n}} d y \\
& \widetilde{\Pi}_{n m}=\int_{-H}^{0} \frac{\tilde{Y}_{n}^{\prime} \tilde{Y}_{m}}{\hat{\beta}_{n}} d y \\
& \hat{\Gamma}_{n m}=\int_{-H}^{0} \tilde{Y}_{n} Y_{m} d y \\
& \hat{\Lambda}_{n m}=\int_{-h}^{0} \frac{\tilde{\hat{Y}}_{n}^{\prime} Y_{m}}{\hat{\beta}_{n}} d y \\
& \hat{\Pi}_{n m}=\int_{-h}^{0} \frac{\hat{Y}_{n} Y_{m}}{\hat{\alpha}_{n}} d y
\end{aligned}
$$

and

$$
\widetilde{\hat{Y}}_{m}=\widetilde{Q}_{m} e^{\hat{\beta}_{m}(y+H)}+\widetilde{R}_{m} e^{-\hat{\beta}_{m}(y+H)}
$$

By applying Eqs. (55)-(58), $B_{n}, C_{m}, D_{m}$, and $E_{n}$ can be obtained.

\section{Vertical Wall Modification}

Although viscous effect on the vertical walls of the dike is not so important in considering the dynamic result for structure design such as the drag force acting on the dike, in order to describe the flow kinematics near the vertical walls correctly, viscous flow modifications near the vertical walls are proposed.

It is easy to understand that under the condition of $R \gg 1$ [see Eq. (35)] the above viscous wave solution can be treated as equivalent to Fig. 2 sketchmatically. Therefore, by adding a vertical wall modification indicated as Fig. 3, we may have a com- plete viscous wave solution, which well approximates the real viscous boundary layer shown in Fig. 4 schematically as $R$ $\gg 1$.

The modifications $\hat{\varphi}^{(1)}$ and $\hat{\varphi}^{(3)}$ which still satisfy Eq. (11), are used to fulfill no-slip conditions at $x= \pm b / 2$

$$
\begin{gathered}
\phi_{y}^{(1)}-\hat{\varphi}_{x}^{(1)}=0, \quad-h \leqslant y \leqslant-H \\
\phi_{y}^{(3)}-\hat{\varphi}_{x}^{(3)}=0, \quad-h \leqslant y \leqslant-H
\end{gathered}
$$

Hence, the solution form concerning the modification of vertical wall boundary layer is suggested to be

$$
\begin{aligned}
\hat{\varphi}^{(1)}(x, y, t)= & \sum_{n=0}^{\infty} F_{n} e^{-i \widetilde{\alpha}_{n}(x+b / 2)}\left[\sinh \left(\alpha_{n} y\right)+P_{n} \cosh \left(\alpha_{n} y\right)\right] \\
& -h \leqslant y \leqslant-H \\
\hat{\varphi}^{(3)}(x, y, t)= & \sum_{n=0}^{\infty} G_{n} e^{i \widetilde{\alpha}_{n}(x-b / 2)}\left[\sinh \left(\alpha_{n} y\right)+P_{n} \cosh \left(\alpha_{n} y\right)\right] \\
& -h \leqslant y \leqslant-H
\end{aligned}
$$

where $\widetilde{\alpha}_{n}^{2}=\alpha_{n}^{2}+k_{\omega}^{2}, \operatorname{Re}\left(\widetilde{a}_{n}\right)>0$. The solutions are easily found as

$$
\begin{gathered}
F_{n}=\left\{\begin{array}{c}
i \frac{\alpha_{n}}{\widetilde{\alpha}_{n}}\left(A_{n} e^{\left(-i b \alpha_{n}\right) / 2}+B_{n} e^{i b \alpha_{n} / 2}\right), \quad n=0 \\
i \frac{\alpha_{n}}{\widetilde{\alpha}_{n}} B_{n} e^{i b \alpha_{n} / 2}, \quad n \geqslant 1
\end{array}\right. \\
G_{n}=i \frac{\alpha_{n}}{\widetilde{\alpha}_{n}} E_{n} e^{i b \alpha_{n} / 2}
\end{gathered}
$$

from Eqs. (66) and (67).

As we may see, the vertical wall modification certainly brings an extra constraint of $R \gg 1$ to Eqs. (24)-(29). However, this constraint is satisfied most of the time because the value of water viscosity usually is very small.

\section{Aspects of Numerical Computation}

\section{Wave Numbers}

Looking back on the dispersion relation (40), we can see that it reveals that the first line of the equation is the same as the conventional dispersion relation (6), the second line is small due to that $\left|\hat{\alpha}_{n} h\right| \ll 1$, and the remaining is also not significant as $R \gg 1$. Thus the other terms beside the first line could be regarded as the perturbation owing to the viscous effect and the roots of Eq. (40) can be obtained by using the Newton-Raphson method with wave numbers of the potential analysis as the initial guesses when 

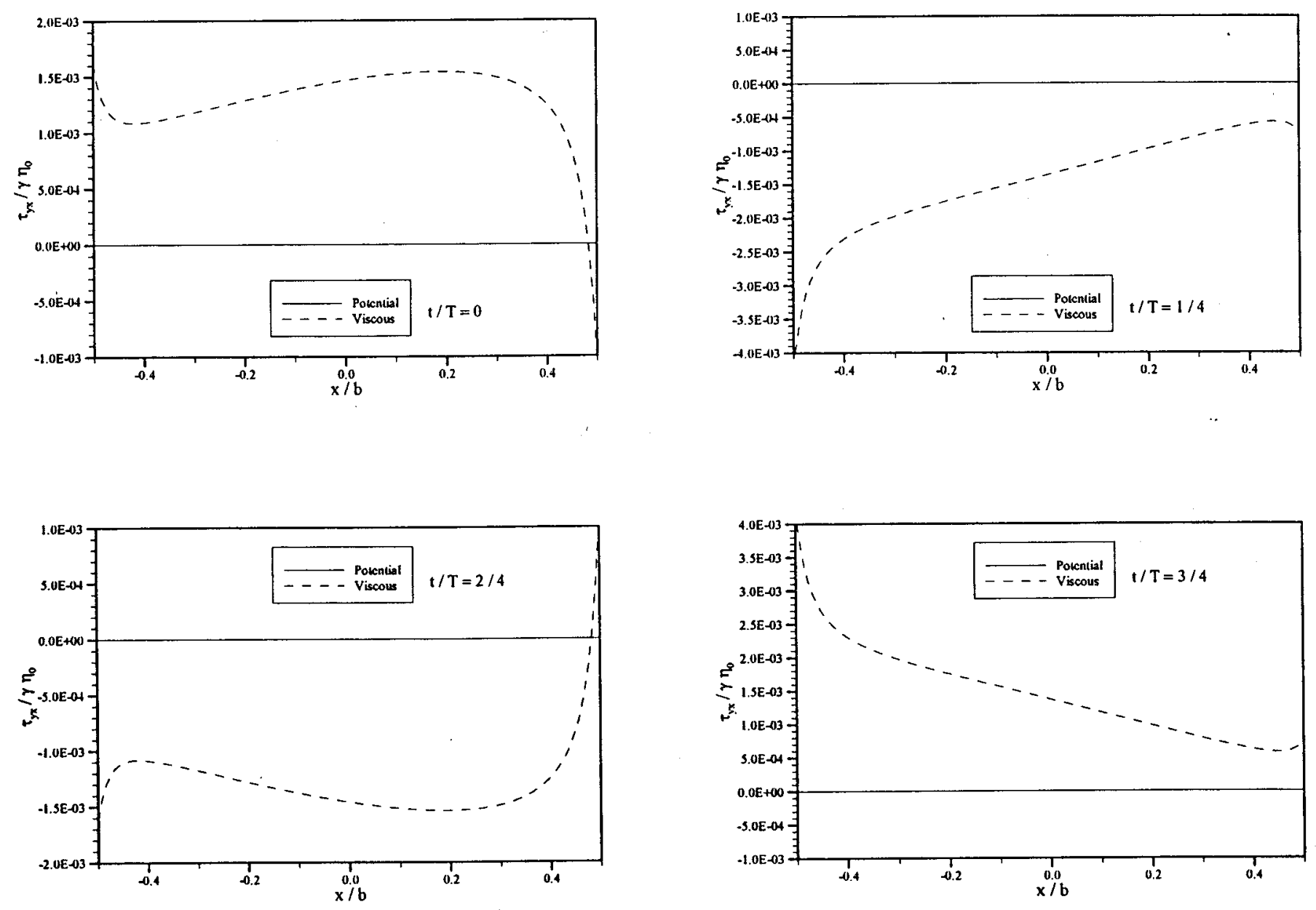

Fig. 15. Shear stress distribution on top of dike $\left(h / H=2, \quad b / H=2, h=0.25^{\mathrm{m}}, \quad \eta_{0}=3.2^{\mathrm{cm}}, \quad T=1.5^{\mathrm{s}}, \quad v=1.17 \times 10^{-6^{\mathrm{m}^{2} / \mathrm{s}}}\right)$

$\mathrm{R} \gg 1$. The relationship of wave numbers between two theories, for the case of $h / H=2, b / H=4, \eta_{0}=3.2^{\mathrm{cm}}$, and $T=1.5^{\mathrm{s}}$, is shown in Fig. 5.

\section{Terms of Infinite Series}

Truncating the summation of an infinite series of each expression for the solution form is needed in numerical works. We adopt the upper bound relation suggested by Mei and Black (1969) as

$$
N \cong M \frac{h}{H}
$$

in which $N$ and $M$ represent the maximum indices of $n$ and $m$ of the series in Eqs. (24)-(29) and in potential solutions Eqs. (1)(3). Numerical tests were made to check the convergence of the results by varying the number of $N$ and $M$, where we obtain an accuracy within $0.01 \%$ while $N \geqslant 20$.

\section{Results and Discussion}

Since the present study is based on small-amplitude water wave theory, some important restrictions such as deep-water condition and linear wave assumption must be held. That is

$$
\alpha_{0} \eta_{0} \ll 1.0
$$

and

$$
U_{r} \ll 1.0
$$

where $U_{r}$ is the well-known Ursell parameter, defined as

$$
U_{r}=\frac{\alpha_{0} \eta_{0}}{\left(\alpha_{0} h\right)^{3}}
$$

Furthermore, although $R \gg 1$ is required for vertical wall modification, the motion is considered as laminar flow only and the occurrence of separation is not allowed in the present study.

The reflection coefficient, $C_{r}=\left|\eta_{r} / \eta_{0}\right|$, is most interesting to previous studies of potential waves. Fig. 6 shows the oscillatory nature of the reflection coefficient resulting from the interaction between the two ends of the obstacle. Our potential model's result agrees with that of Mei and Black (1969). Note that in Mei and Black (1969), variational method is applied, and the original incident wave is split into a couple of symmetric and antisymmetric waves as illustrated by Fig. 7.

The difference of $C_{r}$ between potential analysis and viscous one is presented in Fig. 8. It is clear that $C_{r}$ of the viscous model is a little smaller but very close to the one of the potential model. The reason for the potential model being overestimated is due to the influence of the viscous effect.

The entire velocity field and surface elevation [recall Eqs. (7) and (15)] of potential and viscous models are shown in Figs. 9 and 10. There seems to be no significant kinematic difference between the two analyses. However, viscous effects are obviously pronounced while focusing on the scopes within the boundary 


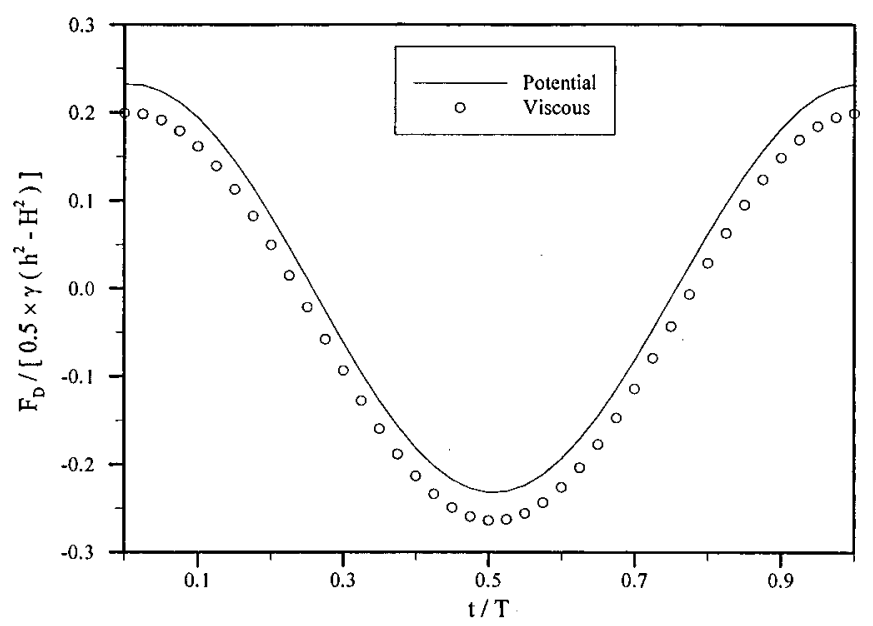

Fig. 16. Variation of drag force within period $(h / H=2, b / H$ $\left.=2, h=0.25^{\mathrm{m}}, \eta_{0}=3.2^{\mathrm{cm}}, T=1.5^{\mathrm{s}}, \quad v=1.17 \times 10^{-6^{\mathrm{m}^{2} / \mathrm{s}}}\right)$

layer as shown in Figs. 11-13. These figures indicate velocity profiles of both potential and viscous models at $x=0$ and at midpoints of vertical walls within the boundary layers. Note that the reference boundary layer thickness here is defined as

$$
\delta=\sqrt{\frac{v}{\omega h \eta_{0}}}
$$

The velocity profiles of the viscous model behave as symmetric curves, which agree with Lamb (1945) and the boundary layer effects are clarified.

Determination of wave forces on the dike plays an important role in structure design of the submerged dike. The distributions of pressures on vertical walls, shear stresses on the top of the dike, and drag forces acting on the dike are investigated. The drag force acting on the dike is contributed mainly by pressure on vertical walls and shear stress on the top of the dike. Fig. 14 indicates pressure distribution [recall Eq. (9)] on vertical walls according to both potential and viscous models. It can be seen from Fig. 14 that pressure distribution is a periodic function of

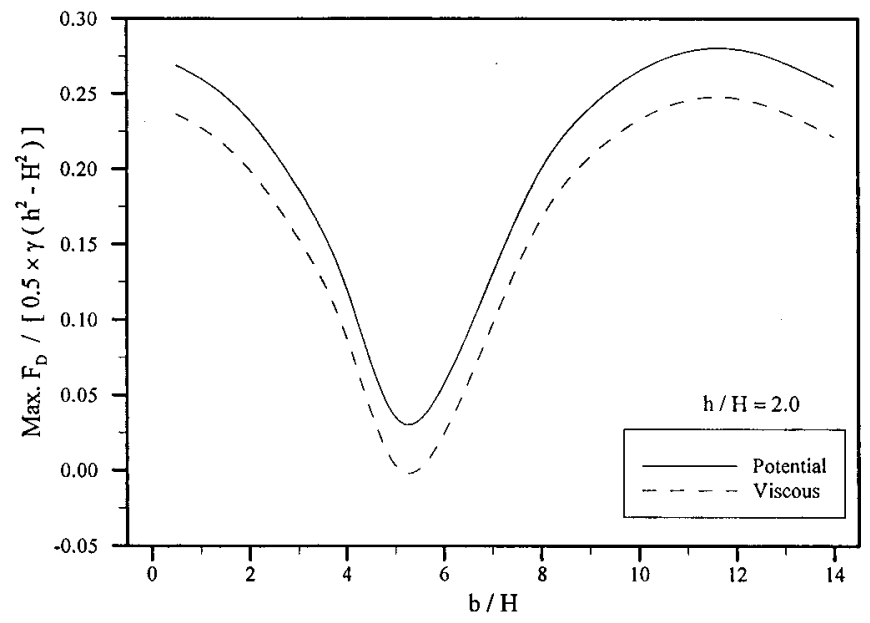

Fig. 17. Maximum drag force at fixed $h / H \quad\left(h=0.25^{\mathrm{m}}, \eta_{0}\right.$ $\left.=3.2^{\mathrm{cm}}, \quad T=1.5^{\mathrm{s}}, \quad \nu=1.17 \times 10^{-6^{\mathrm{m}^{2} / \mathrm{s}}}\right)$

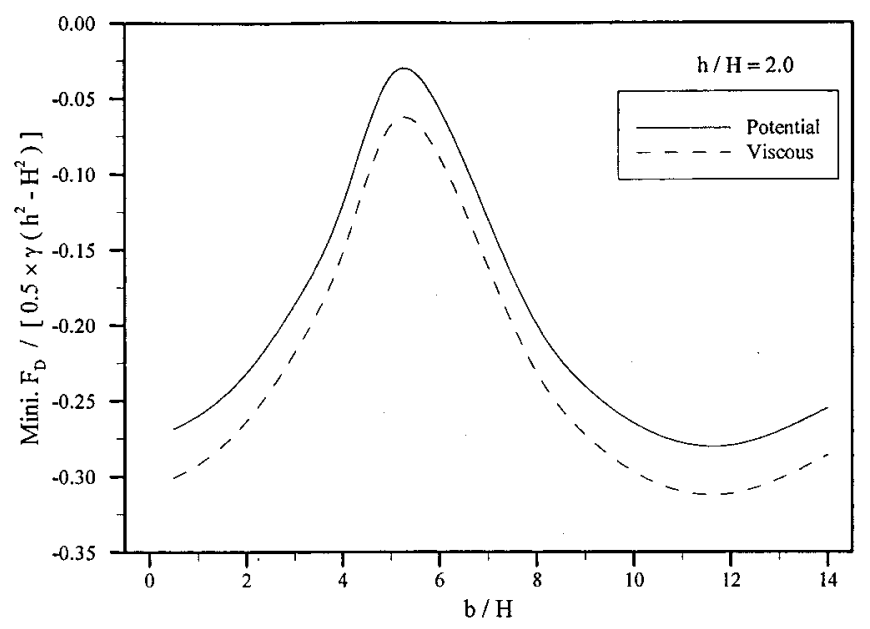

Fig. 18. Minimum drag force at fixed $h / H \quad\left(h=0.25^{\mathrm{m}}, \eta_{0}\right.$ $\left.=3.2^{\mathrm{cm}}, \quad T=1.5^{\mathrm{s}}, \quad v=1.17 \times 10^{-6^{\mathrm{m}^{2} / \mathrm{s}}}\right)$

time, which performs push-and-pull action on the obstacle. Unlike kinematics, the pressure indeed shows deviation between potential and viscous models.

Fig. 15 shows the shear stress, $\tau_{y x}=\mu\left(2 \phi_{x y}-\varphi_{x x}\right.$ $\left.+\varphi_{y y}\right)\left.\right|_{y=-H}$, above the dike. Therefore there is no doubt that this kind of force vanished in the potential model while it is proportional to the increase of the dike width in viscous analysis. Referring to Fig. 12, we can see clearly how the shear stress changes sign corresponding to different flow direction.

Finally, drag force, which is the resultant of the above forces, is presented in Fig. 16. For potential flow

$$
F_{D}=\int_{-h}^{-H} f_{p}^{(L)} d y+\int_{-h}^{-H} f_{p}^{(R)} d y
$$

while for the viscous model

$$
F_{D}=\int_{-h}^{-H}-\sigma_{x x}^{(L)} d y+\int_{-b / 2}^{b / 2} \tau_{y x} d x+\int_{-h}^{-H}-\sigma_{x x}^{(R)} d y
$$

where $f_{p}=$ pressure distribution on the vertical wall; $\sigma_{x x}$ $=$ normal stress on the vertical wall; and $\tau_{y x}=$ shear stress on the top of the dike. (Positive $F_{D}$ points to the right hand side.) We

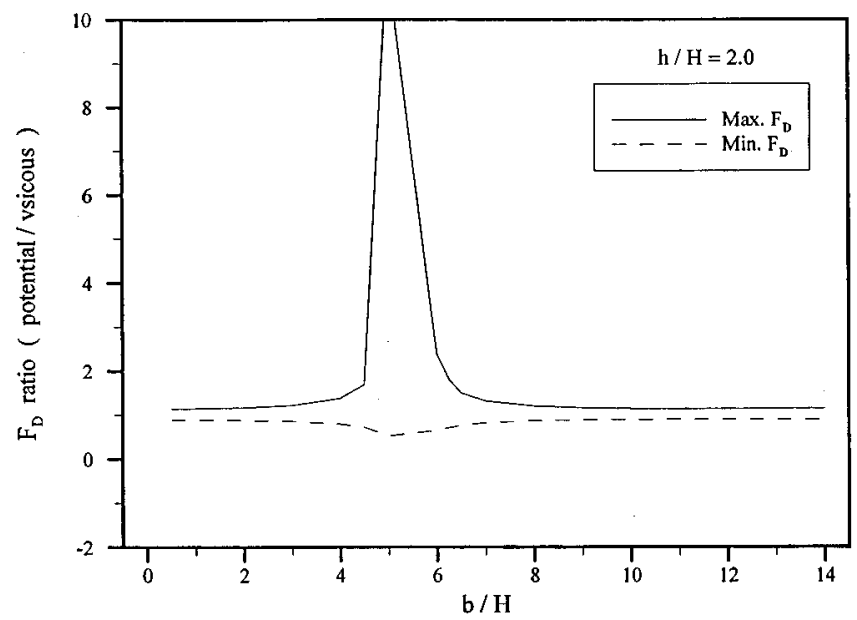

Fig. 19. Ratio of drag force at fixed $h / H \quad\left(h=0.25^{\mathrm{m}}, \eta_{0}\right.$ $\left.=3.2^{\mathrm{cm}}, \quad T=1.5^{\mathrm{s}}, \quad v=1.17 \times 10^{-6^{\mathrm{m}^{2} / \mathrm{s}}}\right)$ 


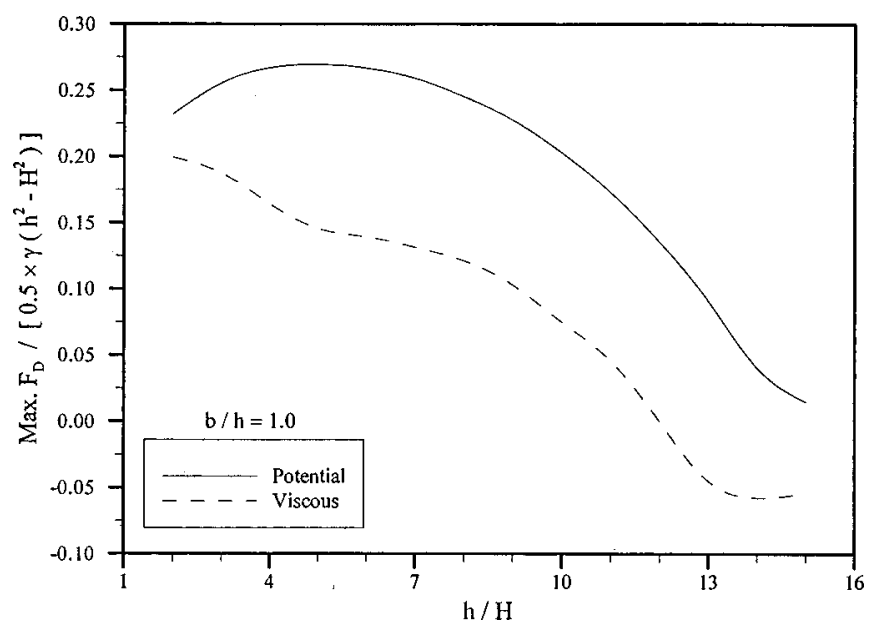

Fig. 20. Maximum drag force at fixed $b / h \quad\left(h=0.25^{\mathrm{m}}, \eta_{0}\right.$ $=3.2^{\mathrm{cm}}, \quad T=1.5^{\mathrm{s}}, \quad \nu=1.17 \times 10^{-6^{\mathrm{m}^{2} / \mathrm{s}}}$ )

may see from Fig. 16 that the potential model holds higher values compared to the viscous model for the maximum (the peak), while it reveals contradictory values for the minimum (the trough).

By varying dike width $b$ or water depth above the dike $H$, the behaviors of extreme drag forces (e.g., the peak and the trough of Fig. 16) are delivered in Figs. 17-22. From Figs. 17 and 18 we find that when $h / H=2$, the potential model always overestimates $\max F_{D}$, while it usually underestimates $\min F_{D}$. When $b / H \approx 5$ $h / H=2$, the ratio between the two models is the biggest as shown in Fig. 19. As $b / h=1$, Figs. 20 and 21 reveal that the potential model in general underestimates $\min F_{D}$ up to about $80 \%$ as $h / H$ becomes larger, while it always overestimates $\max F_{D}$. When $h / H \approx 12 b / h=1$, the potential model even overestimates 90 times $\max F_{D}$. This is because $\max F_{D}$ of the viscous model almost vanishes at the same time. Figs. 17-22 explain clearly that the viscous effect is not negligible when considering the dynamics of the problem.

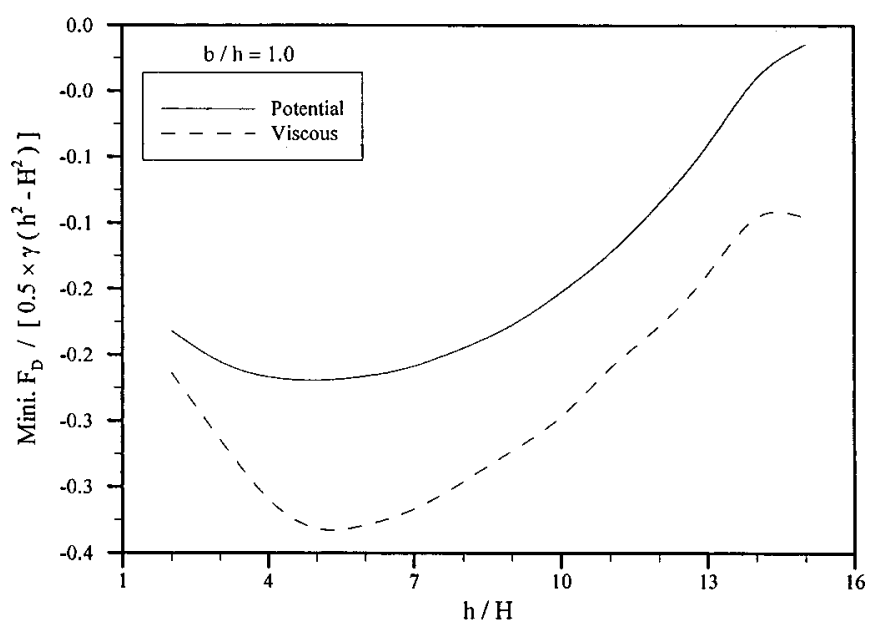

Fig. 21. Minimum of drag force at fixed $b / h\left(h=0.25^{\mathrm{m}}, \eta_{0}\right.$ $\left.=3.2^{\mathrm{cm}}, \quad T=1.5^{\mathrm{s}}, \quad v=1.17 \times 10^{-6^{\mathrm{m}^{2} / \mathrm{s}}}\right)$

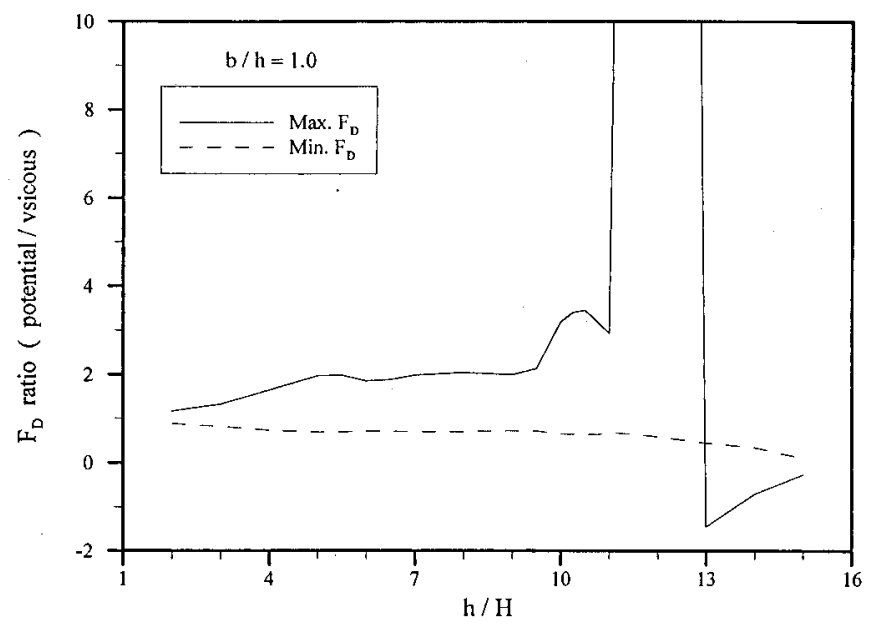

Fig. 22. Ratio of drag force at fixed $b / h \quad\left(h=0.25^{\mathrm{m}}, \eta_{0}\right.$ $\left.=3.2^{\mathrm{cm}}, \quad T=1.5^{\mathrm{s}}, \quad \nu=1.17 \times 10^{-6^{\mathrm{m}^{2} / \mathrm{s}}}\right)$

\section{Conclusions}

In the present study, not only the potential model is solved by the conventional method but also the viscous model is handled by applying newly derived orthogonal inner products. The concept of the orthogonal inner product, which reduces the error of the minimal squares method, is valid in viscous flow. It can be further extended to become a useful mathematical tool.

The result of this study shows that using potential theory to calculate flow kinematics such as reflection coefficients, overall velocity field, etc., are good enough. However, to understand flow dynamics, the viscous effect is not negligible.

The present investigation may provide useful results for experimental comparison. It also gives helpful information in dealing with computation difficulties such as oscillating free surface and near wall grid size.

\section{Acknowledgment}

This research is sponsored by National Science Council of the Republic of China under Grant No. NSC 90-2611-E-002-016.

\section{Notation}

The following symbols are used in this paper:

$A_{0}=$ coefficient for incident wave;

$B_{n}, b_{n}=$ coefficient in general solution for region (1);

$b=$ width of submerged dike;

$C_{m}, c_{m}=$ coefficient in general solution for region (2);

$C_{r}=$ reflection coefficient;

$D_{m}, d_{m}=$ coefficient in general solution for region (2);

$E_{n}, e_{n}=$ coefficient in general solution for region (3);

$\mathrm{F}=$ Froude number;

$F_{D}=$ drag force;

$F_{n}=$ coefficient of vertical wall modification for region (1);

$f_{p}=$ pressure distribution on vertical wall;

$G_{n}=$ coefficient of vertical wall modification for region (3);

$H=$ water depth in region (2); 
$h=$ water depth in regions (1) and (3);

$P=$ perturbed pressure;

$\mathrm{R}=$ Reynolds number;

$T=$ time period;

$U_{r}=$ Ursell parameter;

$\alpha=$ irrotational wave numbers in regions (1) and (3);

$\hat{\alpha}=$ horizontal boundary layer thickness factor in regions (1) and (3);

$\widetilde{\alpha}=$ boundary layer thickness factor of vertical wall modification;

$\beta=$ irrotational wave numbers in region (2);

$\hat{\beta}=$ boundary layer thickness factor in region (2);

$\delta=$ boundary layer thickness;

$\eta=$ free surface displacement;

$\eta_{0}=$ amplitude of incident water wave;

$\eta_{r}=$ reflection wave amplitude in region (1);

$\nu=$ kinematic viscosity;

$\rho=$ density of fluid;

$\sigma_{i j}=$ perturbed stress;

$\tau_{i j}=$ shear stress;

$\phi=$ irrotational potential;

$\varphi=$ rotational potential; and

$\omega=$ angular frequency.

\section{References}

Aris, R. (1962). Vectors, tensors and the basic equations of fluid mechanics, Prentice-Hall, Englewood Cliffs, N.J.

Lamb, H. (1945). Hydrodynamics, 6th Ed., Cambridge University Press, Cambridge, U.K.

Lo, E. Y. M., and Lee, W. K. (2000). "Wave scattering by a surface piercing membrane of finite extent." Proc., 4th Int. Conf. on Hydrology, Yokohama, Japan, 717-722.

Mei, Chiang C., and Black, Jared L. (1969). "Scattering of surface waves by rectangular obstacles in waters of finite depth." J. Fluid Mech., 38, 499-511.

Miles, John W. (1967). "Surface-wave scattering matrix for shelf." $J$. Fluid Mech., 28, 755-767.

Morse, P. M., and Feshbach, H. (1978). Methods of theoretical physics, McGraw-Hill, New York.

Newman, J. N. (1965). "Propagation of water waves past long twodimensional obstacles." J. Fluid Mech., 23, 23-29.

Ou Yang, H. T., Huang, L. H., and Hwang, W. S. (1997). “The interface of a semi-submerged obstacle on the porous breakwater." Appl. Ocean Res., 19, 263-273.

Rhodes-Robinson, P. F. (1979). "On surface waves in the presence of immersed vertical boundaries II." Q. J. Mech. Appl. Math., 32, 125133.

Sahoo, T., Yip, T. L., and Chwang, A. T. (2000). "Effect of forces on wave characteristics for a floating elastic plate." Proc., 4th Int. Conf. on Hydrology, Yokohama, Japan, 205-210. 